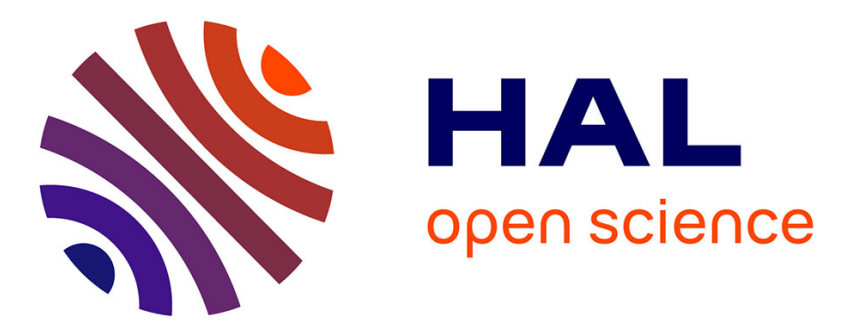

\title{
Co-Cross-Polarization Coherence Over the Sea Surface From Sentinel-1 SAR Data: Perspectives for Mission Calibration and Wind Field Retrieval
}

\author{
N. Longepe, A.A. Mouche, Laurent Ferro-Famil, R. Husson
}

\section{- To cite this version:}

N. Longepe, A.A. Mouche, Laurent Ferro-Famil, R. Husson. Co-Cross-Polarization Coherence Over the Sea Surface From Sentinel-1 SAR Data: Perspectives for Mission Calibration and Wind Field Retrieval. IEEE Transactions on Geoscience and Remote Sensing, 2022, 60, pp.1-16. 10.1109/TGRS.2021.3055979 . hal-03195860

\author{
HAL Id: hal-03195860 \\ https://hal.science/hal-03195860
}

Submitted on 21 Apr 2021

HAL is a multi-disciplinary open access archive for the deposit and dissemination of scientific research documents, whether they are published or not. The documents may come from teaching and research institutions in France or abroad, or from public or private research centers.
L'archive ouverte pluridisciplinaire HAL, est destinée au dépôt et à la diffusion de documents scientifiques de niveau recherche, publiés ou non, émanant des établissements d'enseignement et de recherche français ou étrangers, des laboratoires publics ou privés. 


\title{
Co-Cross-Polarization Coherence Over the Sea Surface From Sentinel-1 SAR Data: Perspectives for Mission Calibration and Wind Field Retrieval
}

\author{
Nicolas Longépé ${ }^{\circledR}$, Alexis A. Mouche ${ }^{\circledR}$, Laurent Ferro-Famil ${ }^{\circledR}$, Member, IEEE, and Romain Husson
}

\begin{abstract}
Spaceborne synthetic aperture radar (SAR) has been used for years to estimate high-resolution surface wind field from the ocean surface backscattered signal. Current SAR platforms have one single fixed antenna, and traditional inversion/retrieval schemes rely on one copolarized channel, leading to an unconstrained optimization problem for providing independent estimates of wind speed and direction. For routine application, this is generally solved with a priori information from the numerical weather prediction (NWP) model, inducing severe limitations for rapidly evolving meteorological systems where discrepancies can be significant between model and measurements. In this study, we investigate the benefit of having two simultaneous acquisitions with phase-preserving information in copolarization and cross polarization provided by Sentinel1 (S-1). A comprehensive analysis of the co-cross-polarization coherence (CCPC) is performed to adequately estimate and calibrate CCPC values from S-1 interferometric wide (IW) mode images acquired over the ocean. A new polarimetric calibration (PolCAL) methodology based on least-squares (LS) criterion and direct matrix inversion is proposed yielding crosstalk estimates. We document CCPC odd symmetry with respect to relative wind direction for light to medium wind speeds (up to $14 \mathrm{~m} / \mathrm{s}$ ) and incidence angle from $30^{\circ}$ to $45^{\circ}$. The azimuthal modulation is found to increase with both wind speed and incidence angle. An analytical model $\mathbf{C}$-band polarimetric geophysical model function (CPGMF) is provided. The synergy of the CCPC with other radar parameters, such as backscattering coefficients or Doppler, to further constrain the inversion scheme is assessed, opening new perspectives for SAR-based wind field retrieval independent of any NWP model information.
\end{abstract}

Manuscript received April 22, 2020; revised August 28, 2020, October 18, 2020, and November 28, 2020; accepted January 8, 2021. This work was supported by the European Space Agency (ESA) through the Scientific Exploitation of Operational Missions (SEOM) "S1 for Ocean Studies" Project. (Corresponding author: Nicolas Longépé.)

Nicolas Longépé was with the Environment and Climate BU, Collecte Localisation Satellites (CLS), 29280 Plouzané, France. He is now with the $\Phi$-Lab Explore Office, European Space Research Institute (ESRIN), European Space Agency (ESA), 00044 Frascati, Italy (e-mail: nicolas.longepe@esa.int).

Alexis A. Mouche is with the Laboratoire d'Océanographie Physique et Spatiale, Institut Francais de Recherche pour l'Exploitation de la Mer, 29280 Plouzané, France.

Laurent Ferro-Famil is with the Remote Sensing Department, Institute of Electronics and Telecommunications of Rennes, University of Rennes 1, 35000 Rennes, France.

Romain Husson is with the Space Observation Division, Environment and Climate BU, Collecte Localisation Satellites (CLS), 29280 Plouzané, France (e-mail: rhusson@groupcls.com).

Color versions of one or more figures in this article are available at https://doi.org/10.1109/TGRS.2021.3055979.

Digital Object Identifier 10.1109/TGRS.2021.3055979
Index Terms-Dual-polarization, polarimetric calibration (PolCAL), Sentinel-1 (S-1), single look complex (SLC), synthetic aperture radar (SAR), wind field retrieval.

\section{INTRODUCTION}

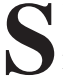
YNTHETIC aperture radar (SAR) ocean surface wind retrieval has been originally based on a single observed quantity, the copolarized normalized radar cross section (NRCS), even though many other SAR observables are potentially available (e.g., cross-polarized NRCS and the Doppler shift). Since the first attempts ([1] with Seasat and [2] with ERS-1) to inverse the NRCS into wind vector, the unique use of the copolarized signal seems to be the rule for routine operational SAR wind measurements. Similarly, the only SAR observable used to provide ocean surface wind fields in the official ESA/Copernicus Level-2 Ocean products is the copolarized NRCS. This approach is directly derived from scatterometry [3] and relies on a transfer function between the radar observables and the surface wind speed and direction. This function, also called the geophysical model function (GMF) or C-band MODel (CMOD) when applied to C-band scatterometer, has been refined several times since the fourth version (CMOD4) [4], [5]. In particular, different strategies have been applied to collocate the radar NRCS with a reference wind given by the ECMWF analysis (CMOD4 [4], [5]), the ECMWF analysis combined with aircraft measurements for high-wind speeds (CMOD5 [6]), or in situ wind measurements (CMODIFR2 [7]). Additional modifications have been also proposed to take into account atmospheric stratification (CMOD5n [8]) or extreme wind (CMOD5h [9]). However, the SAR instruments and missions peculiarities make the challenges of wind measurement very different than for a scatterometer.

First, since existing SARs have one single antenna pointing in the satellite across-track direction, there is only one view angle per wind vector cell. This particularity yields to an underconstrained inverse problem to retrieve both wind speed and direction. The most common method for SAR wind retrieval is to combine copolarized VV NRCS and complementary information on the wind direction as the input of empirical GMFs using a Bayesian scheme [3]. The simplest and probably most efficient method for operational purposes is 
to take the wind direction from a numerical weather prediction (NWP) model. This works generally well for meteorological situations without sharp wind gradients but tends to fail for rapidly evolving systems, such as atmospheric fronts or lowpressure systems, including polar lows and hurricanes. Indeed, for these situations, the global operational NWP models may be too coarse in space and time or may have phasing issues. The direction of the wind (with a $180^{\circ}$ ambiguity) can be also obtained from streak-like features visible in NRCS images and measured using wavelet analysis [10], [11] or local gradients estimation at different spatial scales [12], [13]. This approach mainly relies on the fact that organized large eddies (OLE), also referred to as roll vortices, in the marine atmospheric boundary layer impact the centimeter ocean waves and so the C-band backscattering. However, the occurrence (seasonal and spatial distribution) of the OLE at the global scale as well as the sensitivity of the radar to them are not well characterized yet, leading to questions regarding the feasibility of estimating their directions for all SAR observations. Moreover, although very relevant as an indication of the wind flow, the OLE orientation is not strictly aligned with the ocean surface wind direction. Typically, the measurements from Weather Surveillance Radar by Morrison et al. [14] in the case of hurricane-generated wind rolls indicate that their most probable orientation was tilted by $10^{\circ}$ from the mean wind toward the center. This value is further confirmed by the model experiments from [15].

Second, SAR can provide other observables than a scatterometer, which can help to overcome the limitation of having one single antenna for geophysical applications over the ocean. In particular, the launch of ENVISAT/ASAR in 2002 enabled to measure the geophysical Doppler shift in VV and $\mathrm{HH}$ polarizations. When calibrated, this radar quantity allows ocean surface current radial component [16], [17] and ocean surface wind vector [18] measurements. As shown by Mouche et al. [18], because of the complementary properties of the geophysical Doppler shift, the use of this new radar observable in combination with the copolarized NRCS increases the constraint on the wind inversion scheme. This leads to better wind directions in complex situations, such as atmospheric fronts or low-pressure systems. However, this radar quantity is very sensitive to the accuracy of the attitude orbit control system (AOCS), and its absolute calibration is difficult to achieve. Also, the launch of Radarsat-2 in 2007 enabled to get routine measurements in cross polarization. The weak sensitivity of the cross-polarized NRCS to incidence angle and to wind direction relative to the antenna look angle and the higher sensitivity to wind speed than for copolarization are three key properties of this new polarization [19] that fostered several studies for geophysical applications over the ocean [20]. The most striking is certainly the direct use of the cross-polarized channel for hurricane wind measurements [21], [22]. However, the signal-to-noise ratio (SNR) is very low for the cross-polarized channel, and the noise can be an issue if not properly taken into account. As for Doppler, with this new radar observable, new inversion schemes were proposed to combine $\mathrm{VH}$ and $\mathrm{VV}$ intensities channels [23]. To note, these results have now inspired new scatterometer concepts relying on Doppler and/or VH-NRCS, such as the next MetOp scatterometer [24], [25].

Third, due to the on-board memory limitations, the acquisition strategy, and the multiple SAR acquisition modes, the number of large swath products acquired over the ocean from SAR sensors is far less than for scatterometers. The scatterometer-derived CMOD-based solutions have been systematically applied to C-band SAR: GMFs for VV and a socalled polarization ratio for $\mathrm{HH}$ polarization [26] However, since the launch of ENVISAT/ASAR and Radarsat-2, the amount of data available has significantly increased. The direct consequences are the recent attempts to derive a GMF directly based on SAR measurements (CSARMOD) for both VV- and HH-NRCSs [27]. In addition, a GMF for $\mathrm{HH}$-polarized SAR data, so-called $\mathrm{CMODH}$, has also been developed using collocated ENVISAT ASAR backscatter measurements and ASCAT winds. CMODH is validated by a large number of Radarsat-2 and S-1A/B HH-polarized acquisitions under different wind speeds and buoy observations [28].

Beyond intensities, SAR sensors have also the capabilities to perform spectral analysis for each polarization channel and even to provide relative phase information between polarization channels with dual- or quad-polarization acquisition modes. Indeed, due to the improved spatial resolution, $\mathrm{S}$-1wave mode measurements can further be extended toward shorter scale waves, i.e., within the surface wave equilibrium range. This allows SAR image cross-spectra estimates, including range-traveling intermediate wind waves. The statistical analysis of the spectral energy confirms its sensitivity to both wind speed and wind direction. Comparable to the Doppler estimate, the signed parameter can be used to reduce the wind direction ambiguity in the inversion of high-resolution wind fields from SAR imagery [29], [30]. Regarding the combination of copolarization and cross-polarization channels, theoretical simulations [31], [32] [33] and airborne scatterometer Ku-band observations [34], [35] showed that the correlation between the two channels has odd symmetry with respect to wind direction, a property that could be complementary to the even symmetry of the co-pol NRCS for ocean surface wind vector retrieval. This characteristic was used with quad-pol Radarsat-2 data to remove the wind direction ambiguity using four conditions on the signs of the real and imaginary parts [36]. Hereinafter and in the alternate way of [36], the cocross-polarization coherence parameter is denoted CCPC to stress the combination of cochannel and cross channel. An initial formulation of the polarimetric backscattering coefficient (i.e., the real part of the nonnormalized co-cross correlation noted $\sigma_{x x y y}^{\circ}$ with $x$ and $y$ interchangeably $\mathrm{H}$ and $\mathrm{V}$ ) was proposed in [34]

$$
\begin{aligned}
\hat{\sigma}_{x x x y}(U, \phi, \theta)=\alpha_{1}(U, \theta, x x x y) & \sin (\phi) \\
& +\alpha_{2}(U, \theta, x x x y) \sin (2 \phi)
\end{aligned}
$$

with $\phi$ being the relative wind direction with respect to range direction and $\theta$ the incidence angle. $\alpha_{1}$ and $\alpha_{2}$ are specific functions tuned to $\mathrm{Ku}$-band scatterometer $\left(\theta>40^{\circ}\right)$, assuming constant cross-to-co polarization ratio $\sigma_{X Y} / \sigma_{X X}=$ $-15 \mathrm{~dB}$. In [37], this function (so-called Polarimetric correlation GMF - PGMF) was adapted and tested with C-band 
Radarsat-2 data using CMOD-IFR2 to handle anisotropy modulation but keeping other Ku-band derived parameterization due to the lack of a consistent data set.

This work relies on the new capabilities of S-1SARs. S-1 is a constellation of two European Space Agency (ESA) C-band SAR satellites developed under the Copernicus program. Since the launch of the first satellite Sentinel-1A (S-1A) on April 3, 2014, and now with the second satellite, Sentinel-1B (S-1B) since April 25, 2016, C-band SAR data have been routinely acquired. Two large swath acquisition modes with only dualpolarization $(\mathrm{VV}+\mathrm{VH}$ and $\mathrm{HH}+\mathrm{HV})$ capabilities are available and mostly used over the ocean: the extra-wide (EW) in the Arctic area and the interferometric wide (IW) swath modes in the coastal regions. With respect to previous studies limited by the data number, this study takes benefit of the unprecedented amount of data available due to the unique acquisition strategy and data policy of the Copernicus/ESA S-1 mission. For IW single look complex (SLC) products, the swath coverage is about $250 \mathrm{~km}$ with a pixel spacing of 2.3 (range) $\times 14.1$ (azimuth) $\mathrm{m}$. EW mode images have larger coverage of $410-\mathrm{km}$ swath but with a pixel spacing of only $5.9 \times 19.9 \mathrm{~m}$ [38]. These two modes acquire data from three to five subswaths, respectively, using the TOPSAR imaging technique. Data are acquired in bursts recursively switching the antenna beam among adjacent subswaths. In each burst, the beam is electronically steered in the azimuth forward direction. This technique preserves phase information: the full exploitation of dual-polarimetric SLC data is, thus, possible. To date, although S-1 SARs routinely measure and provide Doppler shifts, they cannot be used for geophysical applications due to calibration issues. These measurements are not used for this study. The quality and the possible use of VH-NRCS for ocean applications have already been reported by Mouche et al. [39], showing that a noise correction is required before using wide swath products. Thus, we focus here on the correlation between copolarization and crosspolarization channels from S-1 phase-preserving TOPS IW data to further illustrate the potential of the correlation between the two channels for ocean surface wind vector retrieval.

The SAR data set used in this work and the applied processing steps are first presented in Section II. It is shown that the co-cross-polarization coherence (CCPC) estimates from dual-pol S-1 are not properly calibrated. This issue is investigated, and a calibration methodology is proposed in Section III. The applicability of these measurements for wind field retrieval is discussed in Section IV. The amplitude and phase values of co-cross-polarization coherence (CCPC) are presented with respect to ocean surface wind speed, relative wind direction, and incidence angle (see Section IV-A). These estimates are then integrated into the Bayesian wind inversion scheme (see Section IV-B). Discussions and conclusions are given in Sections V and VI. In the Appendix, the analytical formulas are provided with their corresponding set of coefficients for the first C-band Polarimetric coherence GMF, further referred to as C-band polarimetric geophysical model function (CPGMF).
TABLE I

Number of S-1 Products Used in This Study

\begin{tabular}{|c|ccccc|}
\hline Product & 2014 & 2015 & 2016 & 2017 & 2018 \\
\hline S-1A & 507 & 626 & 1802 & 2027 & 253 \\
S-1B & 0 & 0 & 102 & 650 & 141 \\
\hline
\end{tabular}

\section{DAta PRocessing}

In this study, the CCPC values are generated from a massive set of S-1 SLC IW VV-HV products, preferred to EW images due to a limited number of items in the processed database. The temporal distribution of the corresponding $5215 \mathrm{~S}-1 \mathrm{~A}$ and $893 \mathrm{~S}-1 \mathrm{~B}$ images is given in I. Note that, from this point on, the polarimetric convention is used hereafter. The term VV-HV is, thus, employed for dual-polarized S-1 sensors with vertical polarized transmit waves and reception in the vertical and horizontal polarizations. This follows the mathematical notation explained in (4) starting from the emitting system on the right-hand side to the reception on the left-hand side. The whole database has been processed by cluster computing in order to reduce the processing time to a few days. Under the usual assumption that the SLC echoes follow a centered complex circular normal distribution, CCPC is computed as the maximum likelihood estimate (MLE) of the normalized correlation coefficient, as follows:

$$
\hat{\rho}_{v o h v}=\frac{\left\langle S_{v v} S_{h v}^{*}\right\rangle}{\sqrt{\left\langle\left|S_{v v}\right|^{2}\right\rangle\left\langle\left|S_{h v}\right|^{2}\right\rangle}}
$$

where $\langle x\rangle \triangleq \sum_{l=1}^{L} x(l)$, with $x(l)$ a realization of $x, L$ the total number of realizations, and $x^{*}$ the complex conjugate of $x$. The independent realizations, called looks, are sampled over regions having a stationary polarimetric response, made of 1462 adjacent resolution cells in azimuth, covering a complete TOPS mode burst extent, and of 2107 cells in the range direction, resulting in nearly 3000000 total looks, located within an approximately square area in ground coordinates, with $12.5-\mathrm{km}$ long sides. The coherence values obtained from the sequentially and independently processed bursts are projected onto a regular geographical grid with $12.5-\mathrm{km}$ spacing, using a nearest-neighbor interpolation approach to avoid additional multilooking. All gridded results are then collocated with the 10-m sea surface wind from ECMWF atmospheric numerical model running at $0.125^{\circ}$ and $1-\mathrm{h}$ resolution.

CCPC products are analyzed jointly with respect to the incidence angle, wind speed, and wind orientation, relative to the radar range direction, and defined so that $\phi=0^{\circ}$ and $\phi=$ $180^{\circ}$ correspond to upwind and downwind situations, respectively. The mean coherence amplitude, averaged over wind orientation, using values sampled every $6^{\circ}$ over $360^{\circ}$ bins, is shown in Fig. 1.

It is found that the CCPC generally increases with the wind speed and depends on incidence angle as well. Prior to any in-depth analysis related to wind speed, this figure highlights some potential issues with S-1 IW measurements.

1) Some nongeophysical artifacts seem to be directly or indirectly linked with IW subswath limits. They appear 


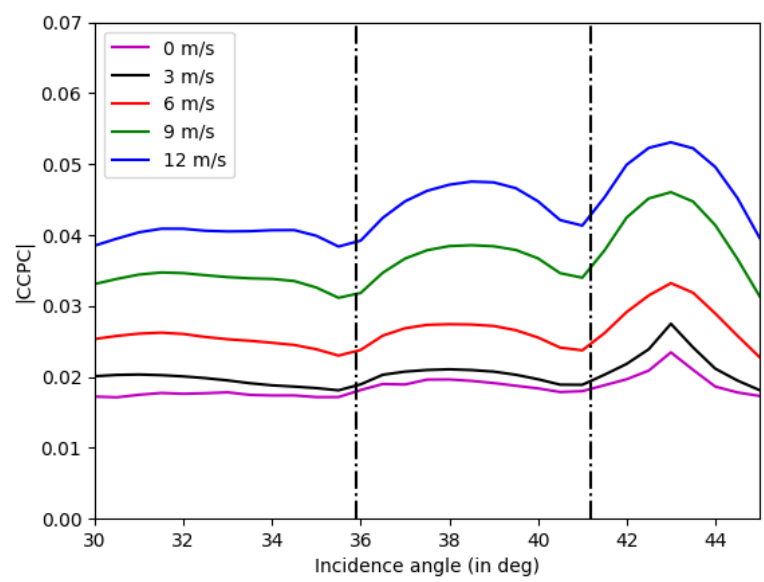

Fig. 1. Estimated CCPC magnitude averaged over all relative wind directions. Vertical dashed lines indicate IW subswath limits.

as a multiplicative effect, whose amplitude increases with the mean coherence value, occurring at the scale of a subswath. No particular geophysical or electromagnetic (EM) scattering reasons could be found to explain such behaviors.

2) Both the real and imaginary parts of the coherence should converge toward zero in the condition of reflection symmetry, supposedly met either at up- or downwind situations, with stable wind condition, or at very low wind situation with no directional preference.

However, Fig. 1 clearly shows an offset of about 0.02 when wind speed is below $3 \mathrm{~m} / \mathrm{s}$.

\section{Proposed S-1 CCPC Calibration TeChnique}

Several kinds of error may affect CCPC estimates, such as the bias of the MLE in (2) [40], the nonstationarity of SLC signals over the sampling regions [41], the decorrelation related to low signal-to-noise power ratio values [42], or signal leakage between vertical and horizontal polarization channels [35].

\section{A. Potential Sources of Error}

In the following, the importance of these sources of error is investigated in the case of CCPC estimation from $\mathrm{S}-1$ data.

1) MLE Bias: The MLE of the level of correlation, $\left|\hat{\gamma}_{h h h o}\right|$, computed from the optimal L-look expression given in (2), is known to be biased, and its expectation is written [40]

$$
\begin{aligned}
\mathrm{E}\left(\left|\hat{\rho}_{v v h v}\right|\right)= & \frac{\Gamma(L) \Gamma(1.5)}{\Gamma(L+0.5)}\left(1-\left|\rho_{v v h v}\right|^{2}\right)^{L} \\
& \times{ }_{3} F_{2}\left(1.5, L, L ; L+0.5,1 ;\left|\rho_{v v h v}\right|^{2}\right) \\
\neq & \left|\rho_{v v h v}\right|
\end{aligned}
$$

where ${ }_{3} F_{2}\left(a_{1}, a_{2}, a_{3} ; b_{1}, b_{2} ; z\right)$ and $\Gamma(z)$ stand for the generalized hypergeometric and Gamma functions, respectively. Fig. 2 shows the plots of the relationship in (2), calculated using Monte Carlo simulations of correlated random sequences, as the numerical evaluation of the hypergeometric function requires extremely intensive computational resources when its arguments, here the number of looks $L$, reach large

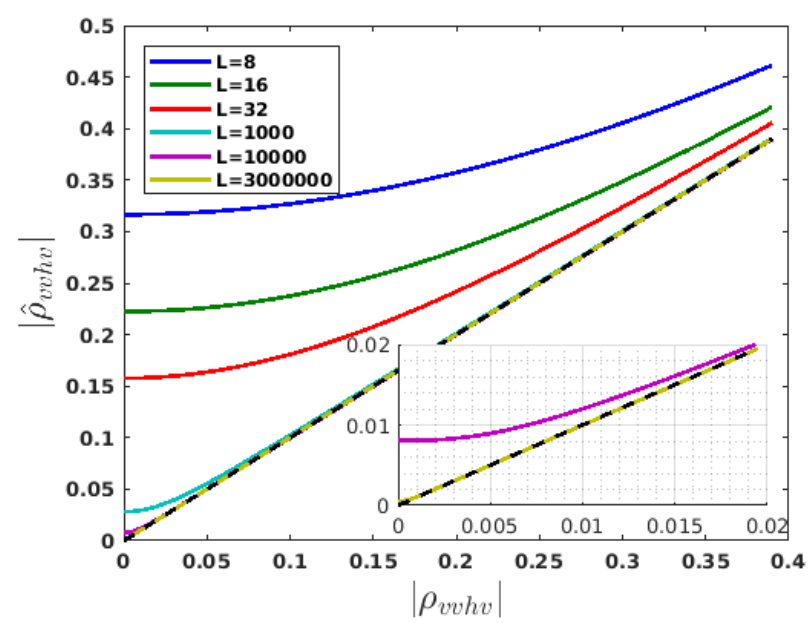

Fig. 2. Mean coherence, $\mathrm{E}\left(\left|\hat{\rho}_{v v h v}\right|\right)$, estimated using (3), as a function of the true value, $\left|\rho_{v v h v}\right|$, for various numbers of looks, L. A zoomed-in view is given for $\left|\rho_{v v h v}\right| \leq 0.02$. The diagonal dashed line represents the locus of unbiased estimates.

values. The bias effect can be clearly perceived from these plots, with $\mathrm{E}\left(\left|\hat{\rho}_{v v h v}\right|\right)$ values departing significantly from the diagonal black dashed line, when an insufficient number of looks, $L$, are used to estimate the low true correlation values $\left|\rho_{v v h v}\right|$. With the wind inversion being generally performed over a $1-\mathrm{km}^{2}$ grid, the CCPC values could be estimated over a similar grid obtained with about $L=10000$ looks. However, given the order of magnitude for the CCPC observed over oceanic scenes using S-1, as displayed in Fig. 1, $L=10000$ looks may reveal insufficient to compute reliable estimates: the bias can be significant for low correlation values. As mentioned in [43], this bias could be compensated using the curve in Fig. 2 as a lookup table, but such an approach would remain imprecise, due to the dispersion of estimates around their mean value, which highly depends on $L$. The plots shown in Fig. 2 indicate that $L=3 \times 10^{6}$ looks, corresponding to $12.5 \mathrm{~km} \times 12.5 \mathrm{~km}$ averaging regions, lead to bias values that remain negligible whatever the true correlation level is.

2) Nonstationary Statistical Behavior: Local phenomena, such as atmospheric or oceanic fronts, and convective rain cells, may lead to heterogeneous features in SAR images acquired over the sea surfaces. The resulting statistical behavior may depart from the Gaussian hypothesis used to derive the MLE in (2) and, hence, affect estimated correlation values. Similar to other factors, such as SAR processing artifacts created by the TOPS acquisition mode along the burst, with the radiometric discontinuity between two adjacent scans, the occurrence of nonstationary phenomena remains limited given the very large amount of images, 6108, processed in this study. These effects are here considered negligible for this study concerned with a massive statistical assessment, as their impact on final estimates remains marginal.

\section{B. Polarimetric Calibration}

As the MLE bias and the nonstationary statistical behavior are discarded as potential sources of error for the CCPC estimates, the decorrelation related to low signal-to-noise

\section{ACCEPTED MANUSCRIPT}


power ratio values [42] and signal leakage between vertical and horizontal polarization channels [35] are jointly analyzed in this section.

According to the available documentation [44], S-1 coand cross-pol channels are independently compensated in amplitude, in order to deliver calibrated $\sigma_{p q}^{\circ}$ values, but are not calibrated or corrected for the relative phase information and potential coupling between the polarimetric channels. In [45], a possible approach is provided to assess the polarimetric distortion matrix of dual-pol S-1 data, including crosstalk, channel imbalance, and the Faraday rotation effects. It assumes the availability of a distributed target having a response with azimuthal symmetry [46], trihedral-like, and oriented dihedral-like targets to retrieve all the parameters. Similar to many polarimetric calibration (PolCAL) techniques, it assumes reflection symmetry [46] over distributed scenes, such as dense forests. As indicated in the introduction, this assumption, despite being widely considered in procedures aiming to detect metallic targets at sea, does not always hold over the sea surface and may be considered as valid only in up- or down-wind situation (wind waves are orthogonal to the range direction) or in case of low wind [31], [35].

1) General Statement for the S-1 Dual-Pol System: The dual-polarization radar system on-board S-1 sensors transmits vertically polarized signals and measures responses in a linear horizontal and vertical polarization basis. In the absence of the Faraday rotation, the received vector can be written as follows [47]:

$$
\left[\begin{array}{l}
M_{h v} \\
M_{v v}
\end{array}\right]=A e^{j \phi}\left[\begin{array}{cc}
1 & \delta_{1} \\
\delta_{2} & f
\end{array}\right]\left[\begin{array}{cc}
S_{h h} & S_{h v} \\
S_{v h} & S_{v v}
\end{array}\right]\left[\begin{array}{c}
\delta_{3} \\
1
\end{array}\right]+\left[\begin{array}{c}
N_{h v} \\
N_{v v}
\end{array}\right]
$$

where the subscripts $h$ and $v$ denote the horizontal and vertical polarizations, respectively, $A$ is the gain factor of the radar system, $e^{j \theta}$ represents a reference round-trip phase shift common to both acquisition channels, and $N_{p q}$ stands for the acquisition white noise, verifying $\mathrm{E}\left(N_{h h} N_{h v}^{*}\right)=\mathrm{E}\left(N_{p q} S_{r s}^{*}\right)=$ $0 \forall p, q, r, s$. Crosstalk parameters are represented by $\delta_{x}$, whereas $f$ is the channel imbalance coefficient. The relationship in (4) may be simplified by considering that crosstalk terms generally have a small amplitude, typically $\left|\delta_{x}\right| \approx$ $-37.4 \mathrm{~dB}$ [48], and quadratic terms may be neglected, as $\left|\delta_{x} \delta_{y}\right| \ll 1$. Accounting for the polarimetric reciprocity, $S_{h v}=$ $S_{v h}$ applying to monostatic radar systems and environmental scenes [49], [50] [51], the expression of the received signal becomes

$$
\begin{aligned}
& M_{h v} \approx S_{h v}+\delta_{3} S_{h h}+\delta_{1} S_{v v}+N_{h v} \\
& M_{v v} \approx f S_{v v}+\left(\delta_{3} f+\delta_{2}\right) S_{h v}+N_{v v}
\end{aligned}
$$

When dealing with calibrated S-1 products acquired over oceanic scenes, one may assume: 1) a unitary channel imbalance factor $f \approx 1$; 2) co-pol channel intensities significantly larger than cross-pol one, $I_{h h}, I_{v v} \gg I_{h v}$; and 3) negligible second-order crosstalk terms. The measured signal intensities can then be formulated as follows:

$$
\begin{aligned}
& \sigma_{v v}^{\circ} \approx I_{v v}+\sigma_{v v}^{n} \\
& \sigma_{h v}^{\circ} \approx \mathrm{E}\left(\left|S_{v h}+\delta_{3} S_{h h}+\delta_{1} S_{v v}\right|^{2}\right)+\sigma_{h v}^{n}
\end{aligned}
$$

with $\sigma_{p q}^{\circ} \triangleq \mathrm{E}\left(\left|M_{p q}\right|^{2}\right), \sigma_{p q}^{n} \triangleq \mathrm{E}\left(\left|N_{p q}\right|^{2}\right)$, and $I_{p q} \triangleq \mathrm{E}\left(\left|S_{p q}\right|^{2}\right)$. In [48], the crosstalk analysis is simply performed using trihedral corner reflectors (TCRs), characterized by a scattering matrix proportional to the identity matrix, $S_{v h}=S_{h v}=0$ and $S_{v v}=S_{h h}=A_{\mathrm{TCR}}$. A function of $\delta_{1}$ and $\delta_{3}$, namely, $\left|\delta_{1}+\delta_{3}\right|$, is estimated from (6) with $\sigma_{0}^{h v}$ and $\sigma_{0}^{v v}$ measured over the TCRs. For all S-1 modes, a value is estimated around $-37.4 \pm 4.7 \mathrm{~dB}[48]$.

2) Proposed Estimation of Crosstalk Parameters: According to the expressions given in (6), the CCPC may be written, before crosstalk compensation, as follows:

$$
\begin{aligned}
\rho_{v v h v} & =\frac{\mathrm{E}\left(M_{v v} M_{h v}^{*}\right)}{\sqrt{\sigma_{v v}^{\circ} \sigma_{h v}^{\circ}}} \\
& \approx \frac{I_{v v h v}+\delta_{3}^{*} I_{v v h h}+\delta_{1}^{*} I_{v v}+\left(\delta_{3}+\delta_{2}\right) I_{h v}}{\sqrt{\sigma_{v v}^{\circ} \sigma_{h v}^{\circ}}}
\end{aligned}
$$

where $I_{p q r s} \triangleq \mathrm{E}\left(S_{p q} S_{r s}^{*}\right)$. In (7), the crosstalk parameters are weighted by the co- and cross-pol intensities, reaching large values compared to $\left|I_{v v h v}\right|$, resulting in nonnegligible undesired terms that are very likely to create a bias. A method is proposed in the following that estimates both the amplitude and phase of all three crosstalk coefficient and then calibrates the CCPC values. To do so, the expression in (7) is simplified by considering only cases with wind conditions for which reflection symmetry holds, i.e., $I_{v v h v}=0$. The second term in the numerator is simplified assuming that over sea surfaces co-pol channel responses are highly correlated with a null phase difference, leading to $I_{v v h h}=I_{v v} / \sqrt{\eta(\theta)}$, with $\eta(\theta)$ being the polarimetric ratio between $\sigma_{0}^{v v}$ and $\sigma_{0}^{h h}$, as defined in [26], which depends on the incidence angle. The expression of $\rho_{v v h v}^{r s}$, the CCPC under the hypothesis of reflection symmetry, can be formulated as follows:

$$
\rho_{v v h v}^{r s}=\frac{\left(\delta_{3}^{*} \beta(\theta)+\delta_{1}^{*}\right) I_{v v}+\left(\delta_{3}+\delta_{2}\right) I_{h v}}{\sqrt{\sigma_{v v}^{\circ} \sigma_{h v}^{\circ}}}
$$

where $\beta(\theta) \triangleq 1 / \sqrt{\eta(\theta)}$. Crosstalk parameters may then be estimated by minimizing a least-squares (LS) criterion as follows:

$$
\left(\hat{\delta}_{1}, \hat{\delta}_{2}, \hat{\delta}_{3}\right)=\underset{\delta_{1}, \delta_{2}, \delta_{3}}{\operatorname{argmin}} \sum_{m=1}^{M}\left|\hat{\rho}_{v v h v}(m)-\rho_{v v h v}^{r s}\left(\delta_{1}, \delta_{2}, \delta_{3} ; m\right)\right|^{2}
$$

where $\hat{\rho}_{v v h v}(m)$ represents an L-look CCPC estimate computed according to (2), over a local area satisfying the reflection symmetry conditions, and $M$ represents the total number of points in the database for which this assumption is valid. For each image, the modeled CCPC of $(8), \rho_{v v h v}^{r s}\left(\delta_{1}, \delta_{2}, \delta_{3} ; m\right)$, is evaluated by computing $\hat{\eta}(\theta)$ as specified in [26] and intensities approximated as $\hat{I}_{p q}(m) \approx \hat{\sigma}_{p q}^{\circ}(m)-\hat{\sigma}_{p q}^{n}(m)$. The expression in (8) may be rewritten under a linear form by considering separately its real and imaginary components

$$
\left[\begin{array}{c}
\Re\left(\rho_{v h h v}^{r s}\right) \\
\Im\left(\rho_{v v h v}^{r s}\right)
\end{array}\right]=\left[\begin{array}{cc}
\mathbf{b}_{\Re}^{T} & \mathbf{0} \\
\mathbf{0} & \mathbf{b}_{\Im}^{T}
\end{array}\right]\left[\begin{array}{l}
\boldsymbol{\delta}_{\Re} \\
\boldsymbol{\delta}_{\Im}
\end{array}\right]
$$

with $\delta_{\mathcal{X}}=\mathcal{X}(\boldsymbol{\delta})$ and $\delta=\left[\delta_{1}, \delta_{2}, \delta_{3}\right]^{T}$ being the vector of unknown values. The linear transformation is defined by two 
real vectors given by

$$
\begin{aligned}
& \mathbf{b}_{\Re}^{T}=\left[I_{v v}, I_{h v}, I_{h v}+I_{v v} \beta(\theta)\right] / \sqrt{\sigma_{v v}^{\circ} \sigma_{h v}^{\circ}} \\
& \mathbf{b}_{\Im}^{T}=\left[-I_{v v}, I_{h v}, I_{h v}-I_{v v} \beta(\theta)\right] / \sqrt{\sigma_{v v}^{\circ} \sigma_{h v}^{\circ}} .
\end{aligned}
$$

The estimation of $\boldsymbol{\delta}$ using a set of $M$ adequate configurations may be performed by minimizing the linear LS criterion given by

$$
\begin{aligned}
& \left(\widehat{\boldsymbol{\delta}}_{\mathfrak{R}}, \widehat{\boldsymbol{\delta}}_{\Im}\right) \\
& =\underset{\boldsymbol{\delta}_{\Re}, \boldsymbol{\delta}_{\Im}}{\operatorname{argmin}} \sum_{m=1}^{M}\left\|\left[\begin{array}{c}
\Re\left(\hat{\rho}_{v v h v}(m)\right) \\
\Im\left(\hat{\rho}_{v v h v}\right)(m)
\end{array}\right]-\left[\begin{array}{cc}
\widehat{\mathbf{b}}_{\Re}^{T}(m) & \mathbf{0} \\
\widehat{\mathbf{0}} & \widehat{\mathbf{b}}_{\Im}^{T}(m)
\end{array}\right]\left[\begin{array}{l}
\boldsymbol{\delta}_{\Re} \\
\boldsymbol{\delta}_{\Im}
\end{array}\right]\right\|_{2}^{2}
\end{aligned}
$$

The criterion may be rewritten as

$$
\widehat{\boldsymbol{\delta}}=\underset{\boldsymbol{\delta}}{\operatorname{argmin}}\|\widehat{\boldsymbol{\rho}}-\widehat{\mathbf{A}} \boldsymbol{\delta}\|_{2}^{2}
$$

with

$$
\boldsymbol{\delta}=\left[\begin{array}{l}
\boldsymbol{\delta}_{\Re} \\
\boldsymbol{\delta}_{\Im}
\end{array}\right], \quad \widehat{\boldsymbol{\rho}}=\left[\begin{array}{l}
\widehat{\boldsymbol{\rho}}_{\Re} \\
\widehat{\boldsymbol{\rho}}_{\Im}
\end{array}\right], \widehat{\mathbf{A}}=\left[\begin{array}{cc}
\widehat{\mathbf{A}}_{\Re} & \mathbf{0}_{M \times 3} \\
\mathbf{0}_{M \times 3} & \widehat{\mathbf{A}}_{\Im}
\end{array}\right]
$$

where $\widehat{\boldsymbol{\rho}}_{\chi}^{T}=\chi\left(\left[\hat{\rho}_{v v h v}(1), \ldots, \hat{\rho}_{v v h v}(M)\right]\right)$ and $\widehat{\mathbf{A}}_{\chi}^{T}=$ $\chi\left(\left[\widehat{\mathbf{b}}_{\chi}(1), \ldots, \widehat{\mathbf{b}}_{\chi}(M)\right]\right)$. A solution to this linear LS problem may be written as

$$
\widehat{\boldsymbol{\delta}}=\widehat{\mathbf{A}}^{\dagger} \widehat{\boldsymbol{\rho}}=\left(\widehat{\mathbf{A}}^{T} \widehat{\mathbf{A}}\right)^{-1} \widehat{\mathbf{A}}^{T} \widehat{\boldsymbol{\rho}} .
$$

One may note here that the LS solution proposed here is generally implemented using stable procedures [52], [53], rather than through a direct matrix inverse, as shown in (15). The proposed estimation approach may be further simplified by taking into account the block-diagonal structure of $\mathbf{A}$, as detailed hereafter

$$
\widehat{\mathbf{A}}^{T} \widehat{\mathbf{A}}=M\left[\begin{array}{cc}
\widehat{\mathbf{R}}_{\mathbf{b}_{\Re}} & \mathbf{0}_{M \times 3} \\
\mathbf{0}_{M \times 3} & \widehat{\mathbf{R}}_{\mathbf{b}_{\Im}}
\end{array}\right]
$$

where $\widehat{\mathbf{R}}_{\mathbf{b} \chi}=\frac{1}{M} \sum_{m=1}^{M} \widehat{\mathbf{b}}_{\chi}(m) \widehat{\mathbf{b}}_{\chi}^{T}(m)$ represents an estimate of the $(3 \times 3)$ sum-of-squares-and-cross-products $(\mathrm{SSCP})$ matrix of $\mathbf{b} \chi$. An alternative implementation of the LS solution that separately estimates the real and imaginary parts of the crosstalk coefficients is given by

$$
\widehat{\boldsymbol{\delta}}_{\chi}=\frac{1}{M} \widehat{\mathbf{R}}_{\mathbf{b} \chi}^{-1} \widehat{\mathbf{A}}_{\chi}^{T} \widehat{\boldsymbol{\rho}}_{\chi} .
$$

The elements of the original IW products database with relative wind direction close to $0 \pm 180^{\circ}$ are gathered into clusters of similar acquisition conditions, by considering ten wind speed classes, from 5 to $14 \mathrm{~m} / \mathrm{s}$, with $1-\mathrm{m} / \mathrm{s}$ wide bins, and 31 incidence angle groups, from $30^{\circ}$ to $45^{\circ}$, with $0.5^{\circ}$ wide bins. For each combination of wind speed and incidence angle values, the estimated quantities $\hat{\sigma}_{p q}^{\circ}, \hat{I}_{p q}$, and $\hat{\rho}_{v v h v}$ ) are averaged, leading to observable vectors of dimensions $M=310$ elements. The retrieved values are found in Table II, considering S-1 swaths altogether or independently.

The estimated quantities have amplitudes close to the one found in [48], $-37.4 \pm 4.7 \mathrm{~dB}$, except for $\delta_{2}$ that cannot be estimated using a TCR, as shown in (5). Cross-talks values retrieved over separate beams are relatively similar. Different antenna configurations from one subswath to the other could
TABLE II

Estimated Cross-Talks Parameters for S-1A/b IW Swath Mode Beams. Amplitude ARe in dB and Phases in Degrees

\begin{tabular}{|c|c|c|c|c|}
\hline Cross-talk & All beam & IW1 & IW2 & IW3 \\
\hline \hline$\hat{\delta}_{1}$ & -43.08 & -42.18 & -50.52 & -46.39 \\
& $\angle-154.65$ & $\angle-92.91$ & $\angle-113.02$ & $\angle-69.99$ \\
\hline$\hat{\delta}_{2}$ & -27.85 & -24.14 & -29.45 & -27.77 \\
& $\angle-128.48$ & $\angle-130.49$ & $\angle-118.46$ & $\angle-115.47$ \\
\hline$\hat{\delta}_{3}$ & -41.00 & -37.07 & -41.28 & -36.95 \\
& $\angle 65.78$ & $\angle 101.93$ & $\angle 116.44$ & $\angle 131.54$ \\
\hline
\end{tabular}
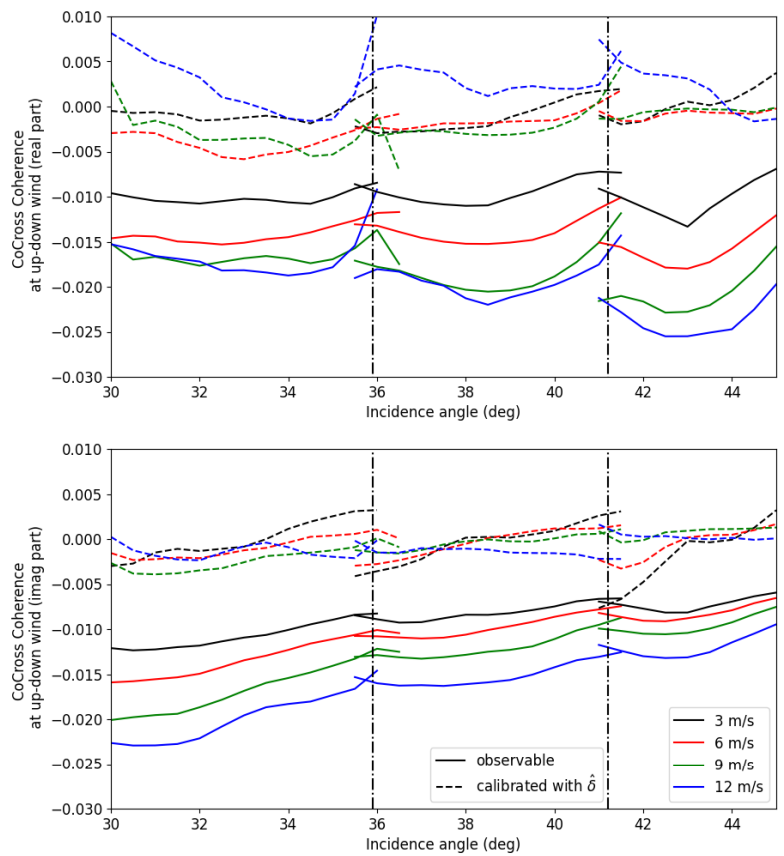

Fig. 3. Co-cross coherence at up- and down-wind conditions where reflection symmetry holds with various wind condition (top: real part and bottom: imaginary part).

explain the observed slight variations, with different antenna configurations of transmit and receive modules.

Once the optimized crosstalk parameters are obtained, the calibrated CCPC can be retrieved from (7), as shown in (18). Note that this process does include the correction for the incoherent noise contribution, as a source of decorrelation

$$
\hat{\rho}_{v v h v}^{\mathrm{cal}}=\frac{\hat{\rho}_{v v h v} \sqrt{\hat{\sigma}_{v v}^{\circ} \hat{\sigma}_{h v}^{\circ}}}{\sqrt{\hat{I}_{v v} \hat{I}_{h v}}}-\frac{\left(\hat{\delta}_{3}^{*} \hat{\beta}(\theta)+\hat{\delta}_{1}^{*}\right) \hat{I}_{v v}+\left(\hat{\delta}_{3}+\hat{\delta}_{2}\right) \hat{I}_{h v}}{\sqrt{\hat{I}_{v v} \hat{I}_{h v}}}
$$

In the case of an up- or down-wind situation, its calibrated values are very close to 0 (less than 0.005 in absolute values for both the imaginary and real parts) (see Fig. 3). The dependence on swath limits seems to be corrected as well. The bias observed in the coherence products is, thus, mostly explained by the noncalibration of S-1 data in terms of polarimetric distortion, including the noise decorrelation that is compensated for.

In the following, the coherence estimates are calculated at a $12.5-\mathrm{km}$ spacing from S-1 IW products thoroughly corrected for the polarimetric distortion and its noise contribution 

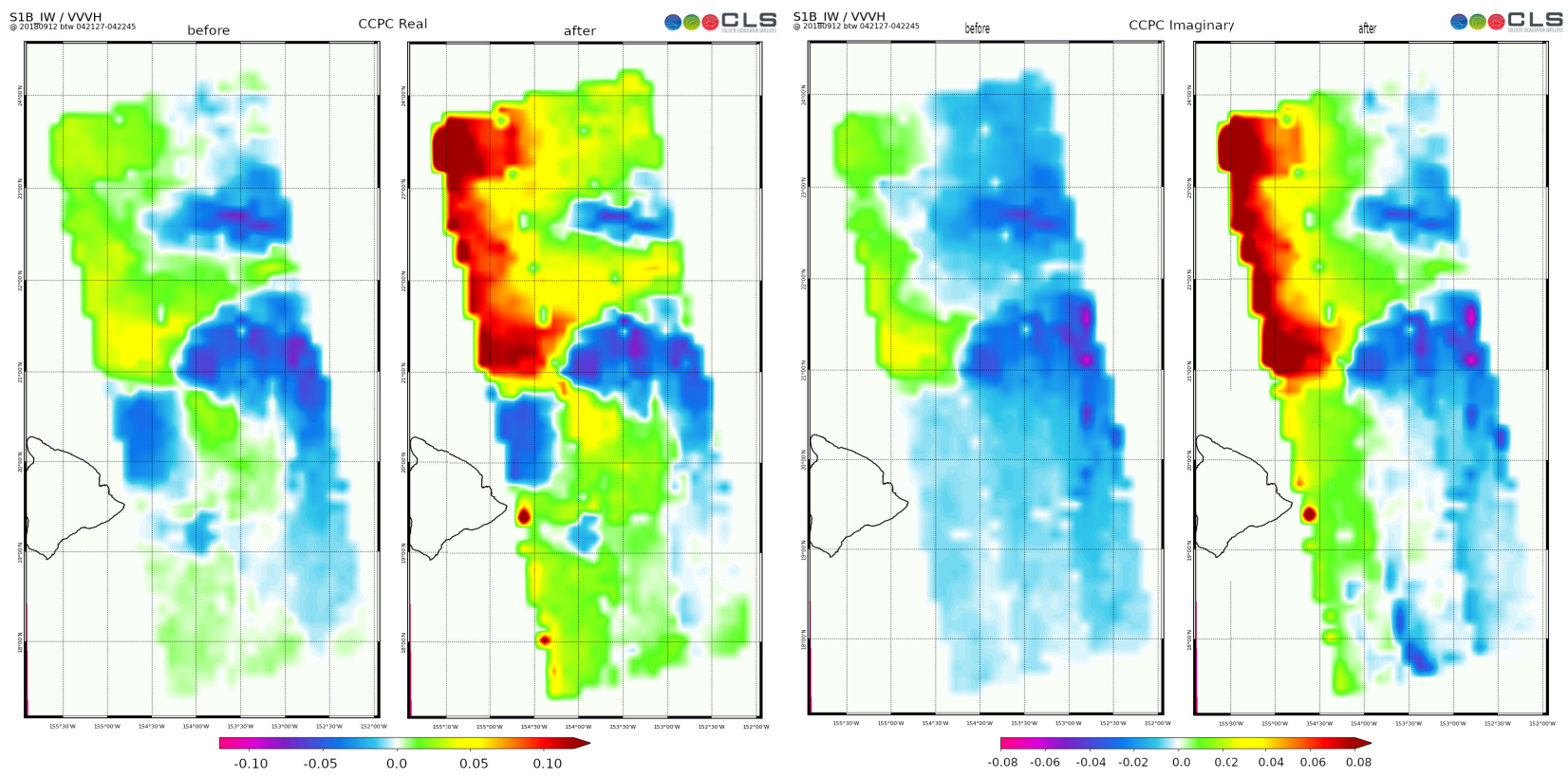

Fig. 4. Case study with S-1B IW data: real (Left) and imaginary (Right) parts of CCPC with before/after the proposed methodology applied. Caution: the sharp edges at the boundary of the SAR images are postprocessing artifacts linked to figure generation.

and collocated with ECMWF ocean surface wind speed and direction. As an illustration, Fig. 4 highlights an example of CCPC information calculated from the IW S-1 products acquired over the tropical cyclone Olivia [here, four slices are stitched (see Fig. 5)]. This case study shows a general situation with wind rotating counterclockwise around the eye with associated speeds not exceeding $10 \mathrm{~m} / \mathrm{s}$ in the southern part and from 10 to $16 \mathrm{~m} / \mathrm{s}$ in the northern part. It is, thus, an ideal study case to illustrate the azimuthal dependence of the CCPC with medium wind force situation. Note here that the position of the eye is slightly shifted between the model and the observation, which can be explained by the modeling errors inherent in this type of situation, but also by the time lag between model outputs and observation time. As indicated in the literature [36], we expect real and imaginary null CCPC values in upwind or downwind situations. Also, the real term should show four different regimes depending on the wind direction (four quadrants are around $-90^{\circ}, 0^{\circ}$ and $90^{\circ}$, and $\pm 180^{\circ}$ ), which is well observed in Fig. 4. The imaginary part should be roughly signed positively and negatively according to the sign of the wind direction; Figs. 6 and 7 will show more details. For areas with a downwind situation where the relative wind direction is close to \pm 180 (in fact above $+135^{\circ}$ or below $-135^{\circ}$ ), the imaginary part remains close to zero (see the southern part of the eye). The amplitude of the CCPC is higher when the wind is strong, as illustrated in the northern part of the eye. At the northeastern part of the eye, around $23^{\circ} N$, there is also a discontinuity clearly visible on the roughness image. While it is not predicted by the model, the real and imaginary parameters of the CCPC well depict this wind field discontinuity. After the proposed methodology is applied, a general positive shift for the real and imaginary components is applied. The signs of the complex CCPC values are more consistent with the theory and the literature with respect to its dependence on relative wind direction. In addition, increased amplitudes are output by the methodology once corrected for the noise decorrelation. As observed in some small "dot" areas
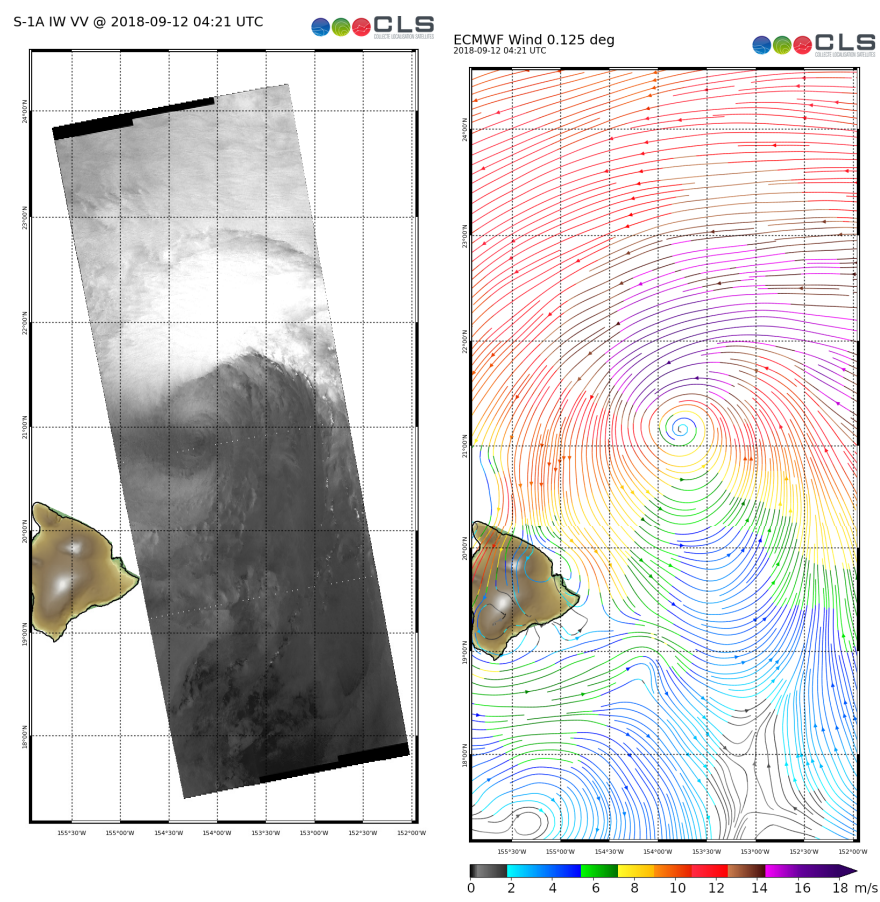

Fig. 5. Case study with S-1B IW data acquired over Hurricane Olivia on 2018/09/12 at 04:21 AM off the coast of Hawaii (USA), ascending pass (Right Looking for S-1) with roughness in co-pol (Left) and ECMWF wind model (Right).

with real CCPC values above 0.10 (see the left-hand side of the image close to Hawaii island), some artifacts appear after the PolCal methodology is applied. They correspond to bright targets, such as vessels. Further investigations are needed to understand the primary reason following (18).

\section{Applicability for Wind Vector Retrieval}

\section{A. Analysis of Co-Cross-Coherence Estimates Over Sea Surface}

Based on the calibrated and collocated CCPC database presented in the previous section, this parameter can now 

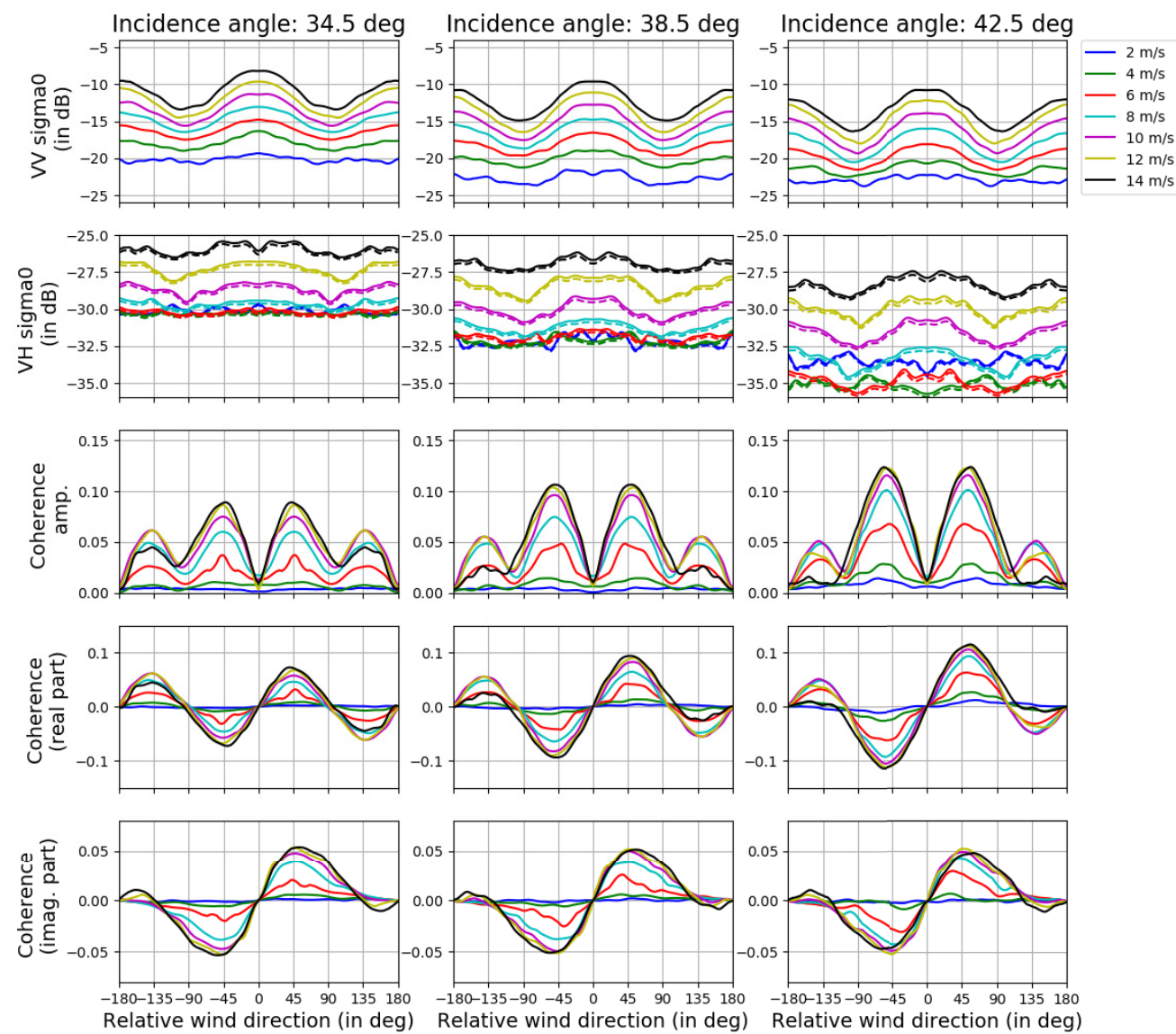

Fig. 6. Analysis of Pol-calibrated NRCS estimates in copolarization (first row) and cross polarization (second row) and CCPC (amplitude and real and imaginary parts, from third to last row) with respect to relative wind direction for different values of wind speed (see color code) and incidence angles: $34.5^{\circ}$ (left), $38.5^{\circ}$ (middle), and $42.5^{\circ}$ (right). The dashed line for $\mathrm{VH}$ sigma0 corresponds to noise-corrected data only, without PolCAL process.

be statistically analyzed with respect to ocean surface wind speed, wind direction relative to the antenna look angle, and the incidence angle. Fig. 6 illustrates the results of this analysis for three different incidence angles located at mid swaths of IW1 (left), IW2 (middle), and IW3 (right), corresponding, respectively, to $34.5^{\circ} \pm 0.5^{\circ}, 38.5^{\circ} \pm 0.5^{\circ}$, and $42.5^{\circ} \pm 0.5^{\circ}$. Each row stands for a different radar parameter (VV and $\mathrm{VH}$ NRCS, and the CCPC terms) plotted as a function of the wind direction relative to the antenna azimuth look angle for different wind speeds ranging from 2 to $14 \mathrm{~m} / \mathrm{s}$. Note that the database does not enable to study the CCPC beyond $14 \mathrm{~m} / \mathrm{s}$ for the entire range of incidence angle and relative wind direction values. The convention of the relative wind direction is such that $0^{\circ}$ stands for upwind configuration, i.e., the wind blows toward the antenna, $180^{\circ}$ stands for downwind configuration, i.e., the wind blows away from the antenna, and $90^{\circ}$ and $90^{\circ}$ stand for crosswind configuration, i.e., the wind blows perpendicular to the antenna.

The following comments can be outlined.

1) The co-pol NRCS variation follows previous welldocumented analysis [27], [54]. A maximum backscattering is obtained in upwind and to a lesser extent in downwind. The backscattering coefficient is also found to be minimum at cross-wind. For a given incidence angle, the averaged (over relative wind direction) backscattering increases with increasing wind speed and for a given wind speed the averaged backscattering decreases with increasing incidence angle.

2) Similar to the copolarization, the analysis of the crosspolarized NRCS estimated from S-1 reveals that, for a given incidence angle, the averaged (over relative wind direction) backscattering increases with increasing wind speed, and for a given wind speed, the averaged backscattering decreases with increasing incidence angle. These results are in line with the most recent studies on the NRCS measured in cross polarization with Radarsat-2 [20] or S-1 [23], [27]. However, we also confirm a slight variation with respect to relative wind direction very similar to copolarization, with maxima obtained for upwind and downwind and minima for crosswind (see [36]). In addition, this azimuthal modulation seems to increase with increasing incidence angle and wind speed. Below $8 \mathrm{~m} / \mathrm{s}$, this modulation is not visible in our analysis. With VV NRCS being much larger than VH NRCS, a remaining leakage from the copolarized signal may induce this modulation in the cross-polarized signal. However, the first order derivation of (6) shows that the terms depending on crosstalk parameters appear to be negligible (see full/dash lines with/without PolCAL process, respectively).

\section{ACCEPTED MANUSCRIPT}



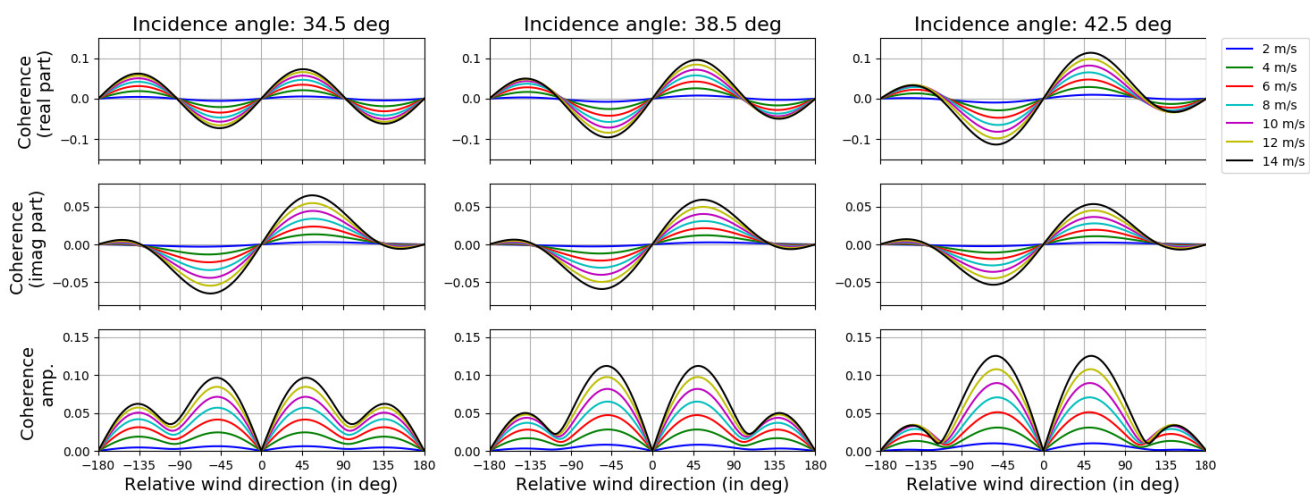

Fig. 7. Analysis of the simulated polarimetric co-cross coherence (amplitude and real and imaginary parts) with respect to relative wind direction and wind speed-at $34.5^{\circ}, 38.5^{\circ}$, and $42.5^{\circ}$.

3) The azimuthal modulations obtained from our analysis for the real and imaginary parts when wind speed is $10 \mathrm{~m} / \mathrm{s}$ are similar to the ones obtained from quadpol RS-2 data achieved by Zhang et al. [36]. An odd symmetry is confirmed with S-1 data. In addition, our data analysis further documents these two quantities. In particular, for a given incidence angle, the azimuthal modulation is found to increase with wind speed and to be negligible for wind speed below $5 \mathrm{~m} / \mathrm{s}$. Then, for a given wind speed, this modulation increases with increasing incidence angle.

4) The amplitude of the coherence also exhibits a clear azimuthal modulation. Like other radar parameters presented here, it also increases with wind speed and incidence angle and becomes negligible for wind speed lower than $5 \mathrm{~m} / \mathrm{s}$.

5) It can be noted that, on the contrary of [36], our analysis indicates that the imaginary part seems not strictly positive in the $[0 ;+180]$ interval (and negative in [ $-180 ; 0]$ interval). This apparent contradiction does not hold for $10-\mathrm{m} / \mathrm{s}$ wind speed, the value at which analysis has been done by Zhang et al. [36].

This data set is further used to build the CPGMF for both real and imaginary CCPC components that allow capturing all the properties discussed in the previous bullets. The analytical formulae with the corresponding set of coefficients and the details regarding the CPGMF accuracy are given in the appendix. It is illustrated in Fig. 7.

\section{B. On the Bayesian Inversion Scheme}

SAR-based wind speed retrieval is generally performed from $\sigma_{0}$ estimates via an inversion of GMF modeling, solving this equation with two unknowns (u-v or U10- $\phi$ wind components) with a priori model information. This minimization problem is often formulated as a Bayesian scheme with independent variables (observation and model) subject to Gaussian uncertainties

$$
\begin{aligned}
J_{\text {ref }}(\mathbf{u}) & =J_{\mathrm{GMF}}(\mathbf{u})+J_{\text {Model }}(\mathbf{u}) \\
& =\left(\frac{\sigma_{0}-\mathrm{GMF}(\mathbf{u})}{\Delta \sigma_{0}}\right)^{2}+\left(\frac{\mathbf{u}_{\text {mod }}-\mathbf{u}}{\Delta \mathbf{u}}\right)^{2}
\end{aligned}
$$

with $\mathbf{u}_{\text {mod }}$ being the a priori wind field associated with $\Delta \mathbf{u}$ error and $\Delta \sigma_{0}$ the NRCS error. The different variables are expected to be spatially correlated [55], [56], but the inversion scheme does not account for it and is applied independently for each wind cell. The zonal and meridional components of the wind vector $\mathbf{u}=\{u, v\}$ are also assumed to be independent. The following additional cost functions for the CCPC and Doppler contributions, respectively, are added in (19). They are defined as

$$
\begin{aligned}
J_{\mathrm{PGMF}}(\mathbf{u})= & \left(\frac{\Re\left(\rho_{v v h v}\right)-\operatorname{PGMF}_{\mathrm{re}}(\mathbf{u})}{\Delta \rho_{\mathrm{re}}}\right)^{2} \\
& +\left(\frac{\Im\left(\rho_{v v h v}\right)-\mathrm{PGMF}_{\mathrm{im}}(\mathbf{u})}{\Delta \rho_{\mathrm{im}}}\right)^{2} \\
J_{\mathrm{CDOP}}(\mathbf{u})= & \left(\frac{f^{\mathrm{DA}}-\mathrm{CDOP}(\mathbf{u})}{\Delta f^{\mathrm{DA}}}\right)^{2} .
\end{aligned}
$$

To note, other SAR-derived parameters, such as the spectral content of the small scales [29], [30], the cross-polarized NRCS, or the OLE orientation derived from image processing could be added in the total cost function. Here, as a first step, copolarized Doppler and the CCPC terms are added to the copolarized NRCS to discuss the complementary of these three parameters. $\mathrm{PGMF}_{\mathrm{re}}$ and $\mathrm{PGMF}_{\mathrm{im}}$ are the real and imaginary parts of the CCPC (for tables or analytic CPGMF functions; see the Appendix). The CDOP function is described in [18]. At this stage, it should be noted that the weighting of the various contributions depends on the chosen uncertainties $\Delta f^{\mathrm{DA}}, \Delta \rho_{\mathrm{re}}$ and $\Delta \rho_{\text {im }}$.

Two uncorrelated sources of uncertainties can be associated with $\Delta \rho$ : the precision of the MLE-based coherence estimate and the quality of the derived PGMFs. The standard deviation of the coherence unbiased estimates is higher than the CramerRao lower bound derived in [57]

$$
E\left(\left|\rho_{v v h v}\right|^{2}\right)>\frac{\left(1-\rho_{v v h v}^{2}\right)^{2}}{2 L}
$$

and shown in Fig. 8. This lower bound may be of interest when designing an adequate inversion scheme: it constrains the minimum error value in the cost function with a link between the spatial resolutions of the native SLC data and the targeted L2 wind product. The quality of the derived PGMF functions 


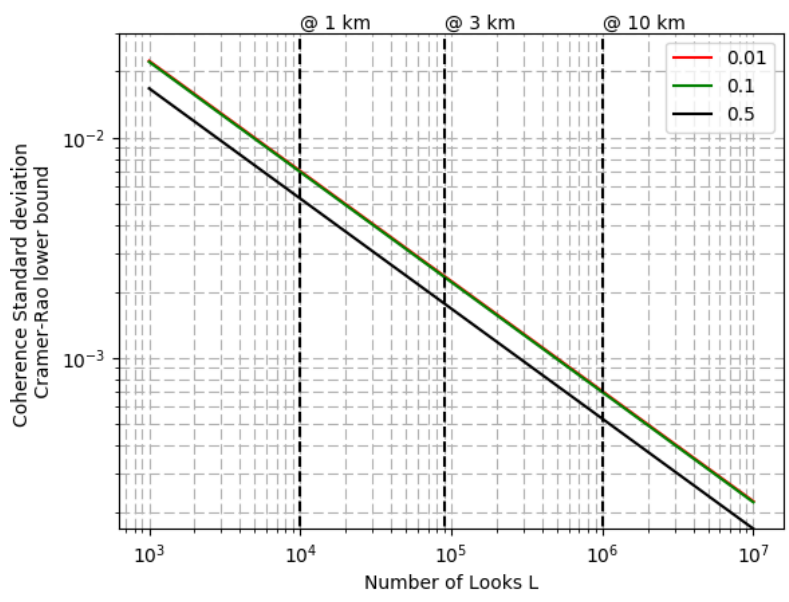

Fig. 8. CR bounds for the standard deviation of the coherence estimate, as function of number of looks L. Vertical lines indicate the corresponding spatial averaging for SLC IW products.

is shown in the Appendix (see Fig. 9). The RMSE of the models against $\mathrm{S}-1$ estimates is in the order of 0.01 and 0.006 for the real and imaginary parts, respectively (about $10 \%$ of the models' magnitude). The remaining noise contamination, the selected analytic functions to model the PGMF, the uncertainties of model wind direction/speed, and, more generally, the nonstationarity and heterogeneities of the data within the 12.5-km cell (see Section III-A2) may be the cause of this variability. In the following, $\Delta \rho=\{0.01,0.006\}$ is, thus, taken for the real and imaginary components of the CCPC.

In Fig. 10, the cost functions for each radar parameter, the ancillary model, and several combinations of these cost functions for various configurations are shown for a given set of measurements and model uncertainties $(\Delta \mathbf{u}=\{\sqrt{3}, \sqrt{3}\}$, $\Delta \sigma_{0}=0.5 \mathrm{~dB}, \Delta f^{\mathrm{DA}}=5 \mathrm{~Hz}$, and $\left.\Delta \rho=\{0.01,0.006\}\right)$. For the first three quantities, they are chosen in line with the literature and close to the current implementation of the ESA S-1 Level-2 processing chain. For the latter, these values are chosen with respect to the measured uncertainties between the models and the data, as explained in the previous paragraph. Measurements are synthesized for a $38.5^{\circ}$ incidence angle, the true wind speed of $7 \mathrm{~m} / \mathrm{s}$, and a wind direction relative to the radar antenna of $45^{\circ}$, using CMOD5 [54] for the NRCS in VV, CDOP [18] for the Doppler in VV, and the PGMFs presented in this study (see the Appendix and Fig. 7). For each of the cost functions presented here, the solution for the zonal and meridional components of the ocean surface wind speed in the space of solutions considered $(\{u, v\})$ is given by the minimum of the cost function (blue).

1) The first row of Fig. 10 presents the cost function obtained when using (a) NRCS $\left(\mathrm{J}_{\mathrm{GMF}}\right)$, (b) the real part of the CCPC $\left(\mathrm{J}_{\mathrm{PGMF}_{\mathrm{Re}}}\right)$, (c) the imaginary part of the CCPC $\left(\mathrm{J}_{\mathrm{PGMF}_{\mathrm{Im}}}\right)$, (d) the two terms of the CCPC $\left(\mathrm{J}_{\mathrm{PGMF}}\right)$, and (e) the Doppler $\left(\mathrm{J}_{\mathrm{CDOP}}\right)$. These cost functions never yields to a unique solution. This illustrates the limitations of an inversion scheme relying on one single radar parameter measured by one single fixed antenna. In the case of $\mathrm{J}_{\mathrm{GMF}}$, the solutions can be found on an ellipse
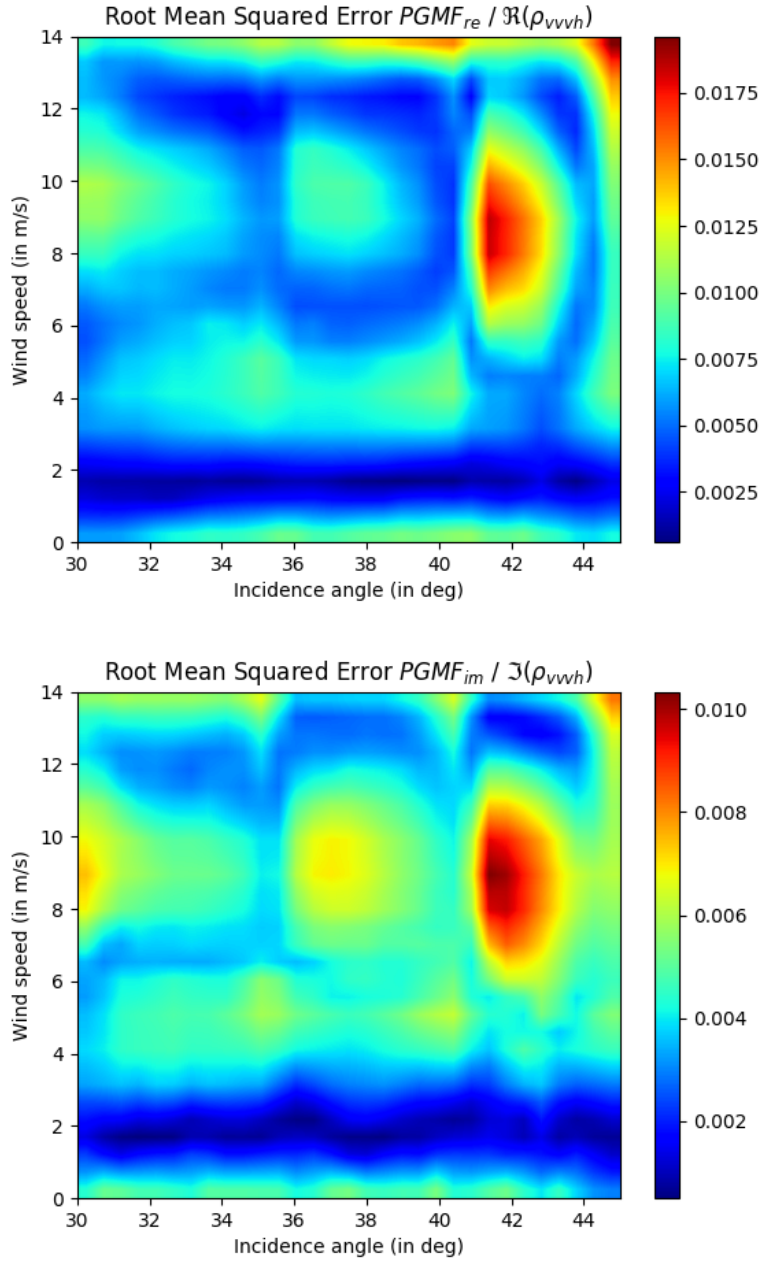

Fig. 9. Root mean square error of $\mathrm{PGMF}_{\mathrm{re}}$ (Top) and $\mathrm{PGMF}_{\mathrm{im}}$ (Bottom) model against averaged coherence estimates for the consolidated database $(121 * 15 * 30) \mathrm{dim}$.

covering all possible wind direction solutions. For $\mathrm{J}_{\mathrm{CDOP}}$, the pattern of the cost function is significantly different with solutions confined to only one half of the space of solutions. Indeed, Doppler is a signed quantity sensitive to the radial (projected along the radar line of sight) component of the wind speed. As observed, $\mathrm{J}_{\mathrm{PGMF}_{\mathrm{Re}}}$ and $\mathrm{J}_{\mathrm{PGMF}_{\mathrm{Im}}}$ are complementary with their respective cost functions minimum located in different areas of the space of solutions. In particular, the real term constrains the solutions with respect to the direction further reduced by the imaginary part (see $\mathrm{J}_{\mathrm{PGMF}_{\mathrm{Re}}}+\mathrm{J}_{\mathrm{PGMF}_{\mathrm{Im}}}$ ).

2) The second row of Fig. 10 illustrates the advantage of using a model as ancillary data to remove the ambiguities. Fig. $10(\mathrm{~g})$ recalls what is the minimization when the only ancillary wind is used in combination with NRCS. This approach is considered as the reference solution here. As already discussed and illustrated by Portabella et al. [3] and Mouche et al. [3], [18], the quality of this solution is strongly driven by the quality of the ancillary data, especially for the wind direction. When the reference approach is further combined with the information from (h) the CCPC, (i) the Doppler in copolarization, or ( $\mathrm{j}$ ) both, the space of possible 

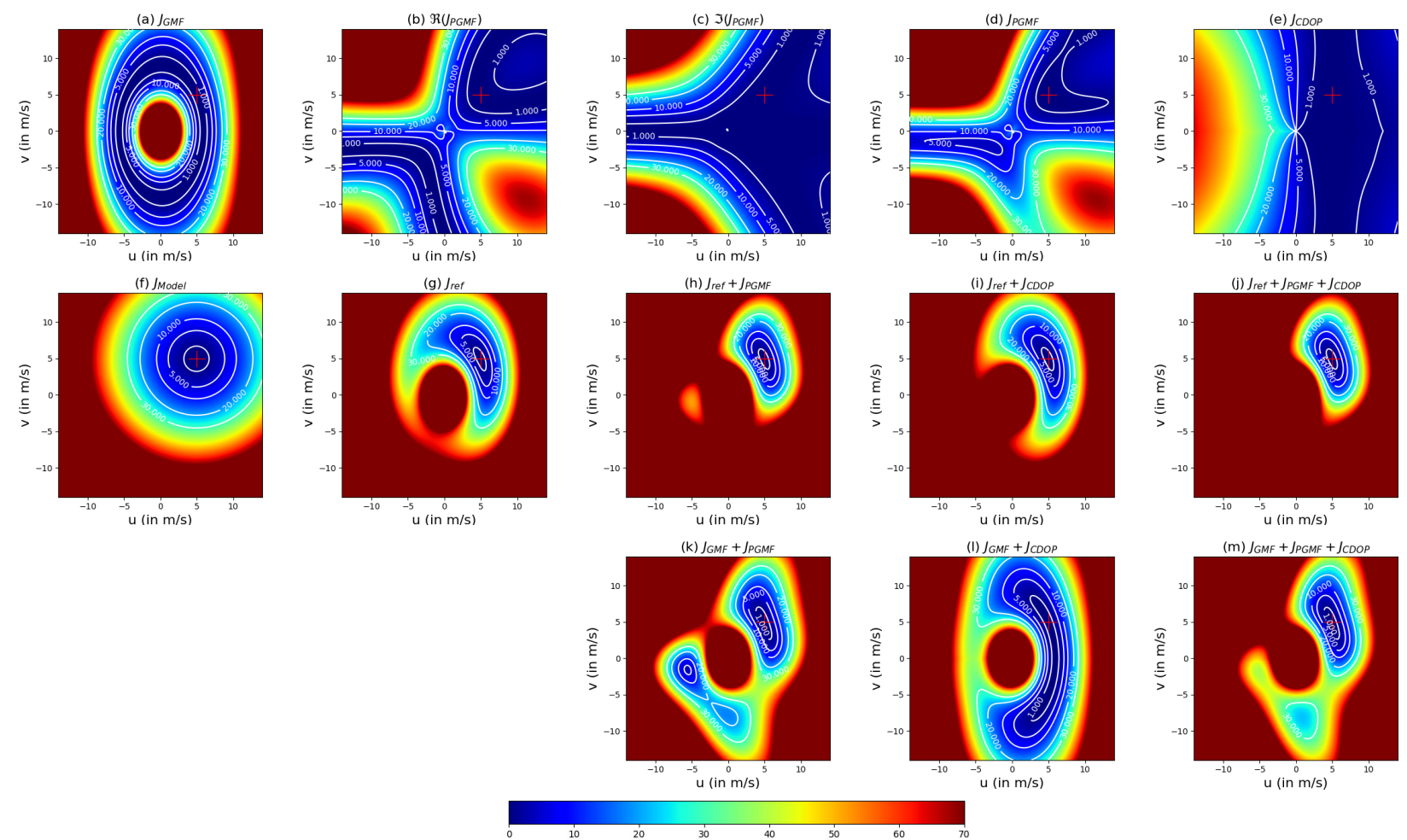

Fig. 10. Cost functions for the incidence angle at $38.5^{\circ}$, the true wind speed at $7 \mathrm{~m} / \mathrm{s}$, and the relative wind direction $45^{\circ}$. The first line corresponds to the independent cost contribution (from Left to Right $J_{\mathrm{GMF}}, J_{\mathrm{PGMF}}$, and $J_{\mathrm{CDOP}}$ ). The second line integrates model $J_{\text {Model }}$ contribution, with various combinations $\left(J_{\text {ref }}, J_{\text {ref }}+J_{\mathrm{PGMF}}, J_{\text {ref }}+J_{\mathrm{CDOP}}\right.$, and $J_{\text {ref }}+J_{\mathrm{PGMF}}+J_{\mathrm{CDOP}}$. The third line does not integrate any model information.

solutions is reduced, showing the interest of adding these radar parameters in the inversion scheme to decrease the weight of the ancillary wind solution in the cost function.

3) With the idea of keeping the inversion scheme independent of any external constraints, the cost functions can be rewritten solely with radar estimates [see Fig. $10(\mathrm{k})-(\mathrm{m})$ ]. Indeed, this approach would prevent any possible wrong information coming from the model in complex meteorological situations. The combinations of Doppler with NRCS and CCPC with NRCS yield to different but overlapping solutions. When all combined, the resulting cost function suggests that these three different and complementary radar parameters could lead to an inversion scheme without external information.

In the aforementioned paragraph, the simulations of the cost functions are computed based on the assumptions that the SAR-based measurements $\left(\sigma_{0}, \Re\left(\rho_{v v h v}\right), \Im\left(\rho_{v v h v}\right)\right.$, and $f^{\text {DA }}$ ) and the model wind information $\left(\mathbf{u}_{\bmod }\right)$ correspond to the actual wind field. In practice, these inputs have a certain degree of variability corresponding to the heterogeneity of sea state conditions within the wind vector cell, the SAR sensor acquisition and imaging processes, and simply model inaccuracies. In order to further evaluate the real potential and limitations of the CCPC in the inversion scheme, a Monte Carlo (MC) approach is tested. A sequence of random offsets given a normal distribution $\mathcal{N}\left(0, \Delta^{2}\right)$ is generated independently for each input. These errors are introduced in the cost functions, and their impacts on the retrieved wind vector are assessed against the true wind field.
In Fig. 11, this assessment is shown for the wind speed at $7 \mathrm{~m} / \mathrm{s}$ and the incidence angle at $38.5^{\circ}$. In this Monte Carlo approach, each radar/model estimate follows a normal distribution with no bias (i.e., centered at its true value from GMF, CDOP, C-PGMF or true wind vector) but with a given variability $(\Delta \mathbf{u}=\{\sqrt{3}, \sqrt{3}\}$ for solid lines or $\Delta \mathbf{u}=\{\sqrt{10}, \sqrt{10}\}$ for dashed lines, $\Delta \sigma_{0}=0.5 \mathrm{~dB}, \Delta \rho=$ $\{0.01,0.006\}$, and $\Delta f^{\mathrm{DA}}=5 \mathrm{~Hz}$ ). RMSEs are given for different wind retrieved variables and cost functions. They are organized in three data sets.

1) Solid lines deal with the addition of radar parameters with respect to the reference $J_{\text {ref }}$ cost function (black solid line) where NRCS is combined with ancillary information from a model. This confirms that the addition of Doppler (green solid line) or CCPC (red solid line) information increases the constraint on the wind inversion and improves performances for both wind speed and direction, with a slight stronger positive impact when the CCPC is considered. When both (i.e., all available information; see solid blue line) are taken into account, the performances on wind direction increase, especially for cross-wind cases.

2) For comparison, other $\mathrm{MC}$ simulations are performed with less accurate model information using $\Delta \mathbf{u}=$ $\{10,10\}$ (dash lines, referred to as $\operatorname{Ref}_{10}$ hereinafter). This intends to simulate an NWP model coarse in space and time, or with phasing issues. This is typically the case for sharp wind gradients and/or rapidly evolving systems, such as atmospheric fronts or low-pressure systems. The quality of $\operatorname{Ref}_{10}$ inversion dramatically

\section{ACCEPTED MANUSCRIPT}



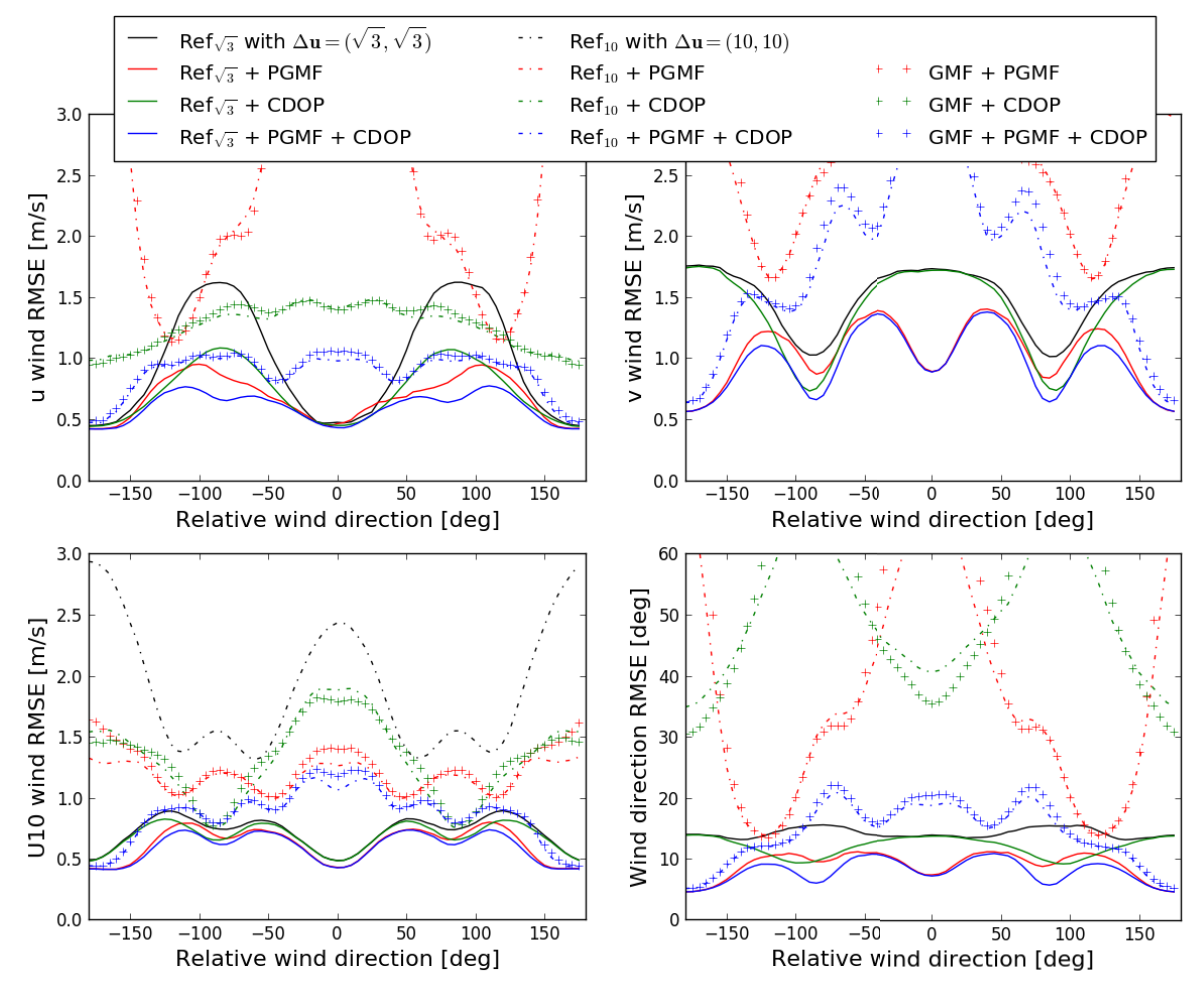

Fig. 11. Monte Carlo simulation and estimation of RMS error on retrieved wind field for true wind speed at $7 \mathrm{~m} / \mathrm{s}$ and various relative wind direction, and the incidence angle at $38.5^{\circ}$. Color lines/styles correspond to various cost functions (see legend).

drops down to about $2 \mathrm{~m} / \mathrm{s}$ for U10 (see black dashed line), being out of the $y$-axis for other wind parameters. Adding Doppler, CCPC, or both radar parameters significantly improves the performances (see red, green, and blue dashed lines).

3) The third set of curves presents the results obtained without any ancillary information. The results are very similar to the one obtained with $\Delta \mathbf{u}=\{10,10\}$. The improved performances achieved when only CCPC (red marker) or Doppler (green marker) is combined with NRCS further exhibits the complementary of these two quantities. As observed, the Doppler mostly helps constraining the wind direction retrieval for situations in the vicinity of up- and down-wind configurations. On the contrary, the CCPC benefits in a crosswind or nearcrosswind wind regimes. This analysis also confirms that the CCPC information is more efficient than Doppler to constrain the wind vector retrieval (see red versus green markers or dash lines). Overall, when the three radar parameters (see blue markers or dash lines) are considered, both wind speed and direction results are improved with moderate-to-good performances (wind speed and direction RMSE of less than $1.2 \mathrm{~m} / \mathrm{s}$ and $20^{\circ}$, respectively).

Provided an accurate estimate of these three radar parameters, their combination with ancillary data remains the best option. However, it appears that the quality of the NWP model still remains decisive. This requires an adequate weighting (and possibly removal) of the a priori model information. If the model contribution is neglected, the use of NRCS with both CCPC and Doppler is mandatory for reasonable results. The CCPC helps constraining the minimization leading to an acceptable estimated wind vector, except for up- or downwind situations, where Doppler information is crucial. All the above discussions apply to wind speed above $5 \mathrm{~m} / \mathrm{s}$ (and up to $14 \mathrm{~m} / \mathrm{s}$ due to our database). For lower wind speed, the general interest of CCPC remains more limited.

\section{Discussion}

To date, the main limitation of SAR systems for ocean surface wind vector retrieval is its single fixed antenna. However, this constrains on the viewing angle can probably be overcome if we take benefit of other SAR capacities. Up until now, when performed routinely or operationally, the ocean wind inversion scheme only relies on the signal intensity in the copolarization channel. However, the last generation of SAR systems provides much more information. Other radar parameters, such as the Doppler and spectral analysis, should be considered. These parameters (including the intensity) need also to be estimated in both copolarization and cross polarization. In addition, the two polarization states can be combined. Here, the estimate and use of the co-cross-polarization coherence term follow this idea. Our work suggests that the use of only three of them could allow not using any ancillary wind information. This could be decisive for the future as the true added value of SAR observations certainly lies in situations where models fail. The on-going work to provide accurate calibrated Doppler from S-1 should lead to a suited data set to test this idea on real data in future work.

\section{ACCEPTED MANUSCRIPT}


With respect to our work, our analysis has to be pursued on more data to extend the range of wind speeds where the CCPC has been analyzed. This is critical for the operational use of such an inversion scheme. In addition, other radar parameters, such as the NRCS in cross polarization (and possibly also the Doppler), will have to be included for ocean surface wind vector retrieval beyond $14 \mathrm{~m} / \mathrm{s}$ and, in particular, for extreme wind speed regimes. In addition, the methodology is built on a database of SLC Level-1 IW products processed with various instrument processing facility (IPF) versions. Homogenization of the input database with enhanced noise annotation (IPF versions newer than v2.9 with both range/azimuth noise dependencies) would be required.

However, probably more challenging would be the derivation of a new inversion scheme to decipher between different geophysical contributions, such as ocean surface current or precipitation, and possibly perform a joint multigeophysical parameters' retrieval. When it comes to the analysis of backscattered signals over the sea surface at very high resolution, this may become nonnegligible. As a matter of fact, a thorough analysis of the cost function minimum should already provide information on areas where other geophysical phenomena impact significantly the radar parameters (or part of them) used in the inversion scheme. Up until now, existing concepts only rely on the use of several antenna [58], [59] when it comes to multigeophysical parameters retrieval with SAR. In any case, the combination of all radar parameters available is certainly the key to maximize constraints on the inversion scheme. In extreme cases, such as areas with strong ocean surface currents or tropical cyclones where both high and complex sea states or intense rainfall can coexist with ocean surface wind, such a multiradar parameters approach could help deciphering between all contributions.

Although promising, such an approach requires an accurate estimate of each radar parameter and its associated uncertainties. To name a few of them, we suggest that: 1) NESZ level shall be even lower (below $-40 \mathrm{~dB}$ ) with estimates in the annotation products of enhanced quality $(\ll \pm 1 \mathrm{~dB})$ and 2) bright targets and any other nongeophysical ocean features shall be filtered out from the CCPC products. One solution could consist in implementing robust coherence estimation techniques, such as those presented in [60]. One may envisage estimating CCPC through advanced speckle filtering techniques, such as the one proposed in [61], which aims to discriminate and process jointly homogeneous regions, whose properties show a sufficient level of similarity.

Concepts similar to the one developed in this study could apply to future spaceborne bistatic missions in either same side-looking interferometric configurations, similar to the one of Tandem-X, or opposite side-looking enabling the measurement of forward scattering contributions (e.g., ESA Earth Explorer 10 Harmony concept [62]), characterized by a significantly improved power budget and additional polarimetric features [63], [64]. On the contrary of the NRCS, the computation and analysis of CCPC from SAR scenes acquired over the ocean are still not widely spread. However, our analysis could be performed with other SAR missions with the possibility to explore frequencies where the contribution of the ocean surface waves to the backscattered signal is different and, thus, to further improve our interpretation of this parameter. For instance, the NRCS analysis of L-band SAR has revealed wind direction dependence significantly different from $\mathrm{C}$-band for wind speeds ranging from 3 to $8 \mathrm{~m} / \mathrm{s}$. In particular, a negative up/crosswind coefficient is observed, indicating more roughness in crosswind for waves scales controlling the backscattering at the L-band [65]. Such differences could lead to a revision of the calibration method and a different behavior of the CCPC at L-band.

\section{Conclusion}

This study takes advantage of the data policy, acquisition strategy, and new capabilities of the S-1 SAR mission with respect to previous European SAR missions to investigate the co- cross-polarization coherence.

In the first part, we showed that the CCPC estimates from S-1 IW data are biased. Under the assumption of reflection symmetry in the up- and down-wind conditions, a PolCal methodology is implemented to correct these biases. Based on the estimate of corrected CCPC over a large data set, we document this parameter as a function of wind direction and incidence angle (between $30^{\circ}$ and $45^{\circ}$ ) for wind speeds ranging from 2 to $14 \mathrm{~m} / \mathrm{s}$. We observe that CCPC amplitude increases with both increasing wind speed and incidence angle. The CCPC amplitude and also the real and imaginary parts exhibit an azimuthal modulation. This modulation increases with wind speed and incidence angle. Moreover, the azimuthal modulation is found to be complementary with both Doppler and NRCS as the maximum/minimum is not reached around up- and down- wind directions. However, both CCPC mean signal and modulation tend to be negligible for wind speeds below $5 \mathrm{~m} / \mathrm{s}$. A simple and analytical formulation is proposed to build a first C-band PGMF for both real and imaginary CCPC components. The corresponding set of coefficients and details regarding CPGMF accuracy are given in the Appendix.

One of the potential interests for using the Doppler centroid anomaly measured by an SAR system over the ocean sea surface was to improve the wind inversion retrieval and, in particular, the wind direction [18]. As a matter of fact, it has been clearly shown that Doppler sensitivity at first order was driven by the ocean surface wind waves rather than the ocean surface current [16], especially for lower incidence angles from $17^{\circ}$ to $30^{\circ}$ [66]. However, as also pointed out by Mouche et al. [18], combining only Doppler with NRCS does not fully constrain the inversion scheme. Based on a developed CPGMF for CCPC and existing GMF for NRCS and CDOP for Doppler in VV polarization, some sensitivity tests are performed to discuss the complementarity of these radar-derived parameters and the necessity of ancillary data to retrieve both wind speed and direction from C-band SAR. Our study shows that the addition of the CCPC significantly increases the constraints. In particular, the complementarity of the three radar quantities allows very similar results to what is achieved when no ancillary data are considered. Such results could dramatically change the whole philosophy of the SAR inversion scheme that heavily relies on ancillary wind direction when SAR wind vectors are produced operationally. However, 
TABLE III

Set of $\alpha$ COEFFICIENTS For S- 1 IW DERIVEd PGMF FUNCTIONS-VALID UP TO $14 \mathrm{~m} / \mathrm{s}$

\begin{tabular}{|c|c|c|c|c|}
\hline$\alpha$ coef. & $\alpha_{1 x}^{r e}$ & $\alpha_{1 x}^{i m}$ & $\alpha_{2 x}^{r e}$ & $\alpha_{2 x}^{i m}$ \\
\hline \hline$x=0$ & $9.75336 \mathrm{e}-5$ & 5.86016 & $9.51124 \mathrm{e}-2$ & $3.87615 \mathrm{e}-1$ \\
$x=1$ & $8.27620 \mathrm{e}-5$ & -4.60297 & $-7.10621 \mathrm{e}-2$ & $-2.29348 \mathrm{e}-1$ \\
$x=2$ & $8.34700 \mathrm{e}-6$ & $2.99795 \mathrm{e}-2$ & $1.80008 \mathrm{e}-3$ & $-2.15936 \mathrm{e}-3$ \\
$x=3$ & -71.4452 & $-1.57449 \mathrm{e}-3$ & $3.97250 \mathrm{e}-1$ & $-1.79613 \mathrm{e}-2$ \\
$x=4$ & 2.14843 & $2.20393 \mathrm{e}-5$ & $-2.67949 \mathrm{e}-2$ & $3.06949 \mathrm{e}-4$ \\
$x=5$ & & & $3.39445 \mathrm{e}-4$ & $-1.93306 \mathrm{e}-6$ \\
\hline
\end{tabular}

such an approach would require at least: 1) some calibrated radar parameters from SAR missions, including Doppler and CCPC; 2) a robust GMF for these two radar parameters with associated errors; and 3) a sufficient amount of data to work with. To note, an independent estimate of the wind direction from SAR (even at a lower resolution than $1 \mathrm{~km}$ ) would also allow a coanalysis of the OLE orientation and wind direction angles that possibly provides information regarding the air-sea interactions in the marine atmospheric boundary layer.

\section{APPENDIX \\ DeRIVATION OF C-BAND PGMF}

The PGMF functions are derived from our compiled database consisting of 6108 IW products with the $L \approx 3 M$ MLEbased coherence estimates colocated with $1 \mathrm{~h}-0.1^{\circ}$ ECMWF products. All the $12.5-\mathrm{km}$ resolution pixels are then averaged with nonoverlapping bins centered from 0 to $14 \mathrm{~m} / \mathrm{s}$ for wind speed with the step of $1 \mathrm{~m} / \mathrm{s}$, from $-180^{\circ}$ to $178.5^{\circ}$ for wind direction with the step of $3^{\circ}$, and from $30^{\circ}$ to $45^{\circ}$ for the incidence angle with the step of $0.5^{\circ}$. From (1), the complex co-cross coherence is formulated as follows:

$$
\begin{aligned}
\rho_{v v h v}(U, \phi, \theta)=( & \left.\alpha_{1}^{\mathrm{re}}(U, \theta)+i \alpha_{1}^{i m}(U, \theta)\right) \sin (\phi) \\
& +\left(\alpha_{2}^{\mathrm{re}}(U, \theta)+i \alpha_{2}^{i m}(U, \theta)\right) \sin (2 \phi)
\end{aligned}
$$

with $\alpha_{1}$ and $\alpha_{2}$, in this first PGMF version, expressed as follows:

$$
\left\{\begin{array}{l}
\alpha_{1}^{\text {re } / \mathrm{im}}=\left(a_{10}+a_{11} U_{10}+a_{12} U_{10}^{2}\right)\left(a_{13}+a_{14} \theta\right) \\
\alpha_{2}^{\text {re } / \mathrm{im}}=\left(a_{20}+a_{21} U_{10}+a_{22} U_{10}^{2}\right)\left(a_{23}+a_{24} \theta+a_{25} \theta^{2}\right)
\end{array}\right.
$$

with $\theta$ in degrees. The results of the optimization processes are found in Table III and are illustrated in Fig. 7.

In a general manner, the accuracy of the co-cross-coherence estimates in our database used to build the PGMF is in the order of $0.005-0.015$ (see Fig. 9). The quality of model fitting varies depending on incidence angle and wind speed. Subswath-related patterns are observed at angles around $36^{\circ}$ and $41^{\circ}$. Further research on dual-polarimetric S-1 data calibration and noise removal might be needed to solve this issue.

\section{ACKNOWLEDGMENT}

The authors would like to thank Dr. Guillaume Hajduch for his advice regarding S-1 quality, Prof. Eric Pottier for his kind suggestion regarding the coherence simulations, and Olivier Archer and Henrick Berger for handling the software environment and deployment at Datarmor facilities.

\section{REFERENCES}

[1] T. W. Gerling, "Structure of the surface wind field from the Seasat SAR," J. Geophys. Res., Oceans, vol. 91, no. 2, pp. 2308-2320, Feb. 1986.

[2] P. W. Vachon and F. W. Dobson, "Validation of wind vector retrieval from ERS-1 SAR images over the ocean," Global Atmos. Ocean Syst., vol. 5, no. 2, pp. 177-187, 1996.

[3] M. Portabella, A. Stoffelen, and J. A. Johannessen, "Toward an optimal inversion method for synthetic aperture radar wind retrieval," J. Geophys. Res., Oceans, vol. 107, no. 8, pp. 1-1-1-13, 2002, doi: 10. 1029/2001JC000925.

[4] A. Stoffelen and D. Anderson, "Scatterometer data interpretation: Estimation and validation of the transfer function CMOD4," J. Geophys. Res., Oceans, vol. 102, no. 3, pp. 5767-5780, Mar. 1997.

[5] A. Stoffelen, "Toward the true near-surface wind speed: Error modeling and calibration using triple collocation," J. Geophys. Res., Oceans, vol. 103, no. 4, pp. 7755-7766, 1998, doi: 10.1029/97JC03180.

[6] H. Hersbach, A. Stoffelen, and S. de Haan, "An improved C-band scatterometer ocean geophysical model function: CMOD5," J. Geophys. Res., vol. 112, no. 3, pp. 1-18, 2007, doi: 10.1029/2006JC003743.

[7] Y. Quilfen, B. Chapron, T. Elfouhaily, K. Katsaros, and J. Tournadre, "Observation of tropical cyclones by high-resolution scatterometry," J. Geophys. Res., Oceans, vol. 103, no. C4, pp. 7767-7786, Apr. 1998, doi: 10.1029/97JC01911.

[8] H. Hersbach, "CMOD5.N: A C-band geophysical model function for equivalent neutral wind," ECMWF Tech. Memoranda, Tech. Rep. 554, Apr. 2008. [Online]. Available: https://www.ecmwf.int/en/elibrary/9873cmod5n-c-band-geophysical-model-function-equivalent-neutral-wind

[9] S. Soisuvarn, Z. Jelenak, P. S. Chang, S. O. Alsweiss, and Q. Zhu, "CMOD5.H-A high wind geophysical model function for C-band vertically polarized satellite scatterometer measurements," IEEE Trans. Geosci. Remote Sens., vol. 51, no. 6, pp. 3744-3760, Jun. 2013.

[10] Y. Du, P. W. Vachon, and J. Wolfe, "Wind direction estimation from SAR images of the ocean using wavelet analysis," Can. J. Remote Sens., vol. 28, no. 3, pp. 498-509, Jan. 2002.

[11] S. Zecchetto, "Wind direction extraction from SAR in coastal areas," Remote Sens., vol. 10, no. 2, p. 261, Feb. 2018.

[12] W. Koch, "Directional analysis of SAR images aiming at wind direction," IEEE Trans. Geosci. Remote Sens., vol. 42, no. 4, pp. 702-710, Apr. 2004.

[13] L. Zhou et al., "An improved local gradient method for sea surface wind direction retrieval from SAR imagery," Remote Sens., vol. 9, no. 7, p. 671, Jun. 2017.

[14] I. Morrison, S. Businger, F. Marks, P. Dodge, and J. A. Businger, "An observational case for the prevalence of roll vortices in the hurricane boundary layer," J. Atmos. Sci., vol. 62, no. 8, pp. 2662-2673, Aug. 2005.

[15] R. C. Foster, "Why rolls are prevalent in the hurricane boundary layer," J. Atmos. Sci., vol. 62, no. 8, pp. 2647-2661, Aug. 2005.

[16] B. Chapron, F. Collard, and F. Ardhuin, "Direct measurements of ocean surface velocity from space: Interpretation and validation," J. Geophys. Res., Oceans, vol. 110, no. 7, pp. 1-17, 2005. [Online]. Available: https://agupubs.onlinelibrary.wiley.com/doi/abs/10.1029/2004JC002809

[17] J. A. Johannessen et al., "Direct ocean surface velocity measurements from space: Improved quantitative interpretation of ENVISAT ASAR observations," Geophys. Res. Lett., vol. 35, no. 22, pp. 1-6, 2008. [Online]. Available: https://agupubs.onlinelibrary.wiley. com/doi/abs/10.1029/2008GL035709

[18] A. A. Mouche et al., "On the use of Doppler shift for sea surface wind retrieval from SAR," IEEE Trans. Geosci. Remote Sens., vol. 50, no. 7, pp. 2901-2909, Jul. 2012.

[19] P. W. Vachon and J. Wolfe, "C-band cross-polarization wind speed retrieval," IEEE Geosci. Remote Sens. Lett., vol. 8, no. 3, pp. 456-459, May 2011.

[20] P. A. Hwang et al., "Cross-polarization geophysical model function for C-band radar backscattering from the ocean surface and wind speed retrieval," J. Geophys. Res., Oceans, vol. 120, no. 2, pp. 893-909, Feb. 2015, doi: 10.1002/2014JC010439.

[21] B. Zhang and W. Perrie, "Cross-polarized synthetic aperture radar: A new potential measurement technique for hurricanes," Bull. Amer. Meteorolog. Soc., vol. 93, no. 4, pp. 531-541, Apr. 2012, doi: 10.1175/ BAMS-D-11-00001.1.

[22] G.-J. van Zadelhoff, A. Stoffelen, P. W. Vachon, J. Wolfe, J. Horstmann, and M. B. Rivas, "Retrieving hurricane wind speeds using crosspolarization C-band measurements," Atmos. Meas. Techn., vol. 7, no. 2, pp. 437-449, Feb. 2014.

\section{ACCEPTED MANUSCRIPT}


[23] A. A. Mouche, B. Chapron, B. Zhang, and R. Husson, "Combined coand cross-polarized SAR measurements under extreme wind conditions," IEEE Trans. Geosci. Remote Sens., vol. 55, no. 12, pp. 6746-6755, Dec. 2017.

[24] M. B. Rivas, A. Stoffelen, and G.-J. van Zadelhoff, "The benefit of HH and VV polarizations in retrieving extreme wind speeds for an ASCATtype scatterometer," IEEE Trans. Geosci. Remote Sens., vol. 52, no. 7, pp. 4273-4280, Jul. 2014.

[25] A. Stoffelen et al., "Scientific developments and the EPS-SG scatterometer," IEEE J. Sel. Topics Appl. Earth Observ. Remote Sens., vol. 10, no. 5, pp. 2086-2097, May 2017.

[26] A. A. Mouche, D. Hauser, J.-F. Daloze, and C. Guerin, "Dualpolarization measurements at C-band over the ocean: Results from airborne radar observations and comparison with ENVISAT ASAR data," IEEE Trans. Geosci. Remote Sens., vol. 43, no. 4, pp. 753-769, Apr. 2005.

[27] A. Mouche and B. Chapron, "Global C-band Envisat, RADARSAT-2 and Sentinel-1 SAR measurements in copolarization and cross-polarization," J. Geophys. Res., Oceans, vol. 120, no. $11, \quad$ pp. $7195-7207,2015 . \quad$ [Online]. Available: http://dx.doi.org/10.1002/2015JC011149

[28] B. Zhang, A. Mouche, Y. Lu, W. Perrie, G. Zhang, and H. Wang, "A geophysical model function for wind speed retrieval from C-band HH-polarized synthetic aperture radar," IEEE Geosci. Remote Sens. Lett., vol. 16, no. 10, pp. 1521-1525, Oct. 2019.

[29] V. Nilsen, G. Engen, and H. Johnsen, "A novel approach to SAR ocean wind retrieval," IEEE Trans. Geosci. Remote Sens., vol. 57, no. 9, pp. 6986-6995, Sep. 2019.

[30] H. Li, B. Chapron, A. Mouche, and J. E. Stopa, "A new ocean SAR cross-spectral parameter: Definition and directional property using the global Sentinel-1 measurements," J. Geophys. Res., Oceans, vol. 124, no. 3, pp. 1566-1577, Mar. 2019. [Online]. Available: https://agupubs.onlinelibrary.wiley.com/doi/abs/10.1029/2018JC014638

[31] S. H. Yueh, R. Kwok, and S. V. Nghiem, "Polarimetric scattering and emission properties of targets with reflection symmetry," Radio Sci., vol. 29, no. 6, pp. 1409-1420, Nov. 1994. [Online]. Available: https://agupubs.onlinelibrary.wiley.com/doi/abs/10.1029/94RS02228

[32] S. H. Yueh, "Modeling of wind direction signals in polarimetric sea surface brightness temperatures," IEEE Trans. Geosci. Remote Sens., vol. 35, no. 6, pp. 1400-1418, Nov. 1997.

[33] A. G. Voronovich and V. U. Zavorotny, "Full-polarization modeling of monostatic and bistatic radar scattering from a rough sea surface," IEEE Trans. Antennas Propag., vol. 62, no. 3, pp. 1362-1371, Mar. 2014.

[34] W.-Y. Tsai, S. V. Nghiem, J. N. Huddleston, M. W. Spencer, B. W. Stiles, and R. D. West, "Polarimetric scatterometry: A promising technique for improving ocean surface wind measurements from space," IEEE Trans. Geosci. Remote Sens., vol. 38, no. 4, pp. 1903-1921, Jul. 2000.

[35] S. H. Yueh, W. J. Wilson, and S. Dinardo, "Polarimetric radar remote sensing of ocean surface wind," IEEE Trans. Geosci. Remote Sens., vol. 40, no. 4, pp. 793-800, Apr. 2002.

[36] B. Zhang et al., "Ocean vector winds retrieval from C-band fully polarimetric SAR measurements," IEEE Trans. Geosci. Remote Sens., vol. 50, no. 11, pp. 4252-4261, Nov. 2012.

[37] Y. Zhang, X. Jiang, Q. Song, M. Lin, and X. Xie, "Coastal wind field retrieval from polarimetric synthetic aperture radar," Acta Oceanologica Sinica, vol. 33, no. 5, pp. 54-61, May 2014, doi: 10.1007/s13131-0140479-5.

[38] M. Bourbigot, H. Johnsen, and R. Piantanida, "ESA Sentinel-1 product definition: 2.7, S1-RS-MDA-52-7440," Eur. Space Agency (ESA), Paris, France, Tech. Rep., Mar. 2016. [Online]. Available: https://sentinel. esa.int/documents/247904/1877131/Sentinel-1-Product-Definition

[39] A. Mouche, B. Chapron, J. Knaff, Y. Zhao, B. Zhang, and C. Combot, "Copolarized and cross-polarized SAR measurements for high-resolution description of major hurricane wind structures: Application to Irma category 5 hurricane," J. Geophys. Res., Oceans, vol. 124, no. 6, pp. 3905-3922, Jun. 2019. [Online]. Available: https://agupubs. onlinelibrary.wiley.com/doi/abs/10.1029/2019JC015056

[40] J.-S. Lee, K. W. Hoppel, S. A. Mango, and A. R. Miller, "Intensity and phase statistics of multilook polarimetric and interferometric SAR imagery," IEEE Trans. Geosci. Remote Sens., vol. 32, no. 5, pp. 1017-1028, May 1994.

[41] M. Jiang, X. Ding, and Z. Li, "Hybrid approach for unbiased coherence estimation for multitemporal InSAR," IEEE Trans. Geosci. Remote Sens., vol. 52, no. 5, pp. 2459-2473, May 2014.
[42] H. A. Zebker and J. Villasenor, "Decorrelation in interferometric radar echoes," IEEE Trans. Geosci. Remote Sens., vol. 30, no. 5, pp. 950-959, Sep. 1992.

[43] R. Touzi, A. Lopes, J. Bruniquel, and P. W. Vachon, "Coherence estimation for SAR imagery," IEEE Trans. Geosci. Remote Sens., vol. 37, no. 1, pp. 135-149, Jan. 1999.

[44] N. Miranda and P. Meadows, "Sentinel-1 radiometric calibration of products," Eur. Space Agency (ESA), Paris, France, Tech. Rep. ESAEOPG-CSCOP-TN-0002, 1.0, 2005. [Online]. Available: https://sentinel. esa.int/documents/247904/685163/S1-Radiometric-Calibration-V1.0.pdf

[45] M. Lavalle, D. Solimini, B. Rosich, and E. Pottier, "Calibration of dual polarimetric C-band SAR data: A possible approach for Sentinel-1," in Proc. PolInSAR Worksho, Frascati, Italy, Jan. 2009, pp. 1-6.

[46] S. V. Nghiem, S. H. Yueh, R. Kwok, and F. K. Li, "Symmetry properties in polarimetric remote sensing," Radio Sci., vol. 27, no. 5, pp. 693-711, Sep./Oct. 1992.

[47] A. Freeman, "Calibration of linearly polarized polarimetric SAR data subject to Faraday rotation," IEEE Trans. Geosci. Remote Sens., vol. 42, no. 8, pp. 1617-1624, Aug. 2004.

[48] P. Meadows, "Sentinel-1 annual performance report 2016," Mission Performance Center, ESA, Tech. Rep. MPC-0366, 2017. [Online]. Available: https://sentinel.esa.int/documents/247904/2370914/Sentinel1-Annual-Performance-Report-2016

[49] L. Tsang and Q. Li, Microwave Remote Sensing Theory. Atlanta, GA, USA: American Cancer Society, 1999. [Online]. Available: https:// onlinelibrary.wiley.com/doi/abs/10.1002/047134608X.W3615

[50] E. C. Koeniguer, "Polarimetric radar images. Signal and image processing," ONERA-French Aerosp. Lab., Palaiseau, France, Tech. Rep., 2014. [Online]. Available: https://tel.archives-ouvertes.fr/tel-01090950

[51] A. Aubry, V. Carotenuto, A. De Maio, and L. Pallotta, "Assessing reciprocity in polarimetric SAR data," IEEE Geosci. Remote Sens. Lett., vol. 17, no. 1, pp. 87-91, Jan. 2020.

[52] C. L. Lawson and R. J. Hanson, Solving Least Squares Problems. Philadelphia, PA, USA: Society Industrial Applied Mathematics, 1995.

[53] P. C. Hansen, Rank-Deficient and Discrete Ill-Posed Problems. Philadelphia, PA, USA: Society Industrial Applied Mathematics, 1998. [Online]. Available: https://epubs.siam.org/doi/abs/10.1137/1.9780898719697

[54] H. Hersbach, "Comparison of C-band scatterometer CMOD5.N equivalent neutral winds with ECMWF," J. Atmos. Ocean. Technol., vol. 27, no. 4, pp. 721-736, Apr. 2010.

[55] Y. He, W. Perrie, Q. Zou, and P. W. Vachon, "A new wind vector algorithm for C-band SAR," IEEE Trans. Geosci. Remote Sens., vol. 43 , no. 7, pp. 1453-1458, Jul. 2005.

[56] H. Shen, W. Perrie, and Y. He, "A new hurricane wind retrieval algorithm for SAR images," Geophys. Res. Lett., vol. 33, no. 21, pp. 1-5, 2006. [Online]. Available: https://agupubs.onlinelibrary.wiley.com/doi/ abs/10.1029/2006GL027087

[57] M. S. Seymour and I. G. Cumming, "Maximum likelihood estimation for SAR interferometry," in Proc. IGARSS-IEEE Int. Geosci. Remote Sens. Symp., vol. 4, Aug. 1994, pp. 2272-2275.

[58] A. C. Martin, C. P. Gommenginger, and Y. Quilfen, "Simultaneous ocean surface current and wind vectors retrieval with squinted SAR interferometry: Geophysical inversion and performance assessment," Remote Sens. Environ., vol. 216, pp. 798-808, Oct. 2018. [Online]. Available: http://www.sciencedirect.com/science/article/pii/S003442571830292X

[59] C. Gommenginger et al., "SEASTAR: A mission to study ocean submesoscale dynamics and small-scale atmosphere-ocean processes in coastal, shelf and polar seas," Frontiers Mar. Sci., vol. 6, p. 457, Aug. 2019 [Online]. Available: https://www.frontiersin.org/article/10.3389/fmars. 2019.00457

[60] G. Shevlyakov and P. Smirnov, "Robust estimation of the correlation coefficient: An attempt of survey," Austrian J. Statist., vol. 40, nos. 1-2, pp. 147-156, Feb. 2016.

[61] C.-A. Deledalle, L. Denis, F. Tupin, A. Reigber, and M. Jager, "NL-SAR: A unified nonlocal framework for resolution-preserving (Pol)(In)SAR denoising," IEEE Trans. Geosci. Remote Sens., vol. 53, no. 4, pp. 2021-2038, Apr. 2015

[62] P. Lopez-Dekker, H. Rott, P. Prats-Iraola, B. Chapron, K. Scipal, and E. D. Witte, "Harmony: An Earth explorer 10 mission candidate to observe land, ice, and ocean surface dynamics," in Proc. IGARSS-IEEE Int. Geosci. Remote Sens. Symp., Jul. 2019, pp. 8381-8384.

[63] D. Comite and N. Pierdicca, "Bistatic radar systems at large baselines for ocean observation," IEEE Trans. Geosci. Remote Sens., vol. 56, no. 3, pp. 1816-1828, Mar. 2018. 
[64] D. Comite and N. Pierdicca, "Monostatic and bistatic scattering modeling of the anisotropic rough soil," IEEE Trans. Geosci. Remote Sens., vol. 57, no. 5, pp. 2543-2556, May 2019.

[65] O. Isoguchi and M. Shimada, "An L-band ocean geophysical model function derived from PALSAR," IEEE Trans. Geosci. Remote Sens., vol. 47, no. 7, pp. 1925-1936, Jul. 2009.

[66] M. J. Rouault, A. Mouche, F. Collard, J. A. Johannessen, and B. Chapron, "Mapping the Agulhas current from space: An assessment of ASAR surface current velocities," J. Geophys. Res., vol. 115, no. 10, pp. 1-14, 2010. [Online]. Available: https://agupubs.onlinelibrary. wiley.com/doi/abs/10.1029/2009JC006050

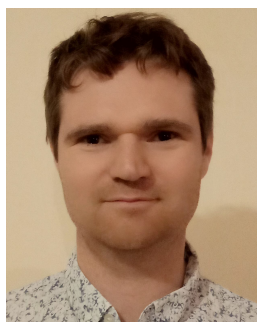

Nicolas Longépé received the M.Eng. degree in electronics and communication systems and the M.S. degree in electronics from the National Institute for the Applied Sciences, Rennes, France, in 2005, and the Ph.D. degree from the University of Rennes I, Rennes, France, in 2008 .

From 2007 to 2010, he was with the Earth Observation Research Center, Japan Aerospace Exploration Agency (JAXA), Tsukuba, Japan. From 2010 to 2020, he was with Collecte Localization Satellites (CLS), Plouzané, France, where he was a Research Engineer with the Space Observation Division. Since September 2020, he has been an Earth Observation Data Scientist at the $\Phi$-Lab Explore Office, European Space Research Institute (ESRIN), European Space Agency (ESA), Frascati, Italy. He has been working on the development of innovative SAR-based applications for environmental and natural resource management (ocean, mangrove, land and forest cover, soil moisture, snow cover, and permafrost) and for maritime security (oil spill, sea ice, iceberg, and ship detection/tracking). His main research interests include (EO) SAR remote sensing and digital technologies, such as machine (deep) learning.

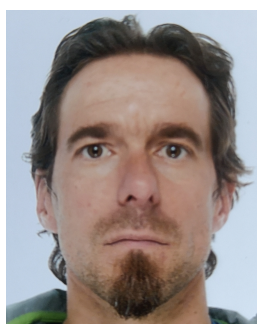

Alexis A. Mouche received the Ph.D. degree in ocean remote sensing from the Université de Versailles Saint-Quentin, Versailles, France, in 2005.

Since 2014, he has been the Senior Research Scientist of the Laboratoire d'Océanographie Physique et Spatiale, Institut Francais de Recherche pour l'Exploitation de la Mer, Plouzane, France. His research interest includes the interaction of electromagnetic and oceanic waves for ocean remote sensing applications.

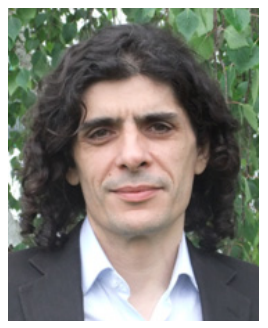

Laurent Ferro-Famil (Member, IEEE) received the Laurea degree in electronics systems and computer engineering and the M.S. and Ph.D. degrees in electronics from the University of Nantes, Nantes, France, in 1996, 1996, and 2000, respectively.

In 2001, he became an Associate Professor with the University of Rennes 1, Toulouse, France, where he has been a Full Professor, since 2011. He is also the Head of the IETR Laboratory, Waves and Signals Department, Rennes, France, and the CESBIO Laboratory, Radar Group, Toulouse. His activities in education are concerned with analog electronics, digital communications, microwave theory, signal processing, and polarimetric synthetic aperture radar (SAR) remote sensing. His research interests include radar signal processing for remote sensing applications, including radar polarimetry theory, electromagnetic imaging, and natural media remote sensing using radar multibaseline PolInSAR data, with application to classification, electromagnetic scattering modeling, physical parameter retrieval, and 3-D reconstruction of environments using tomography.

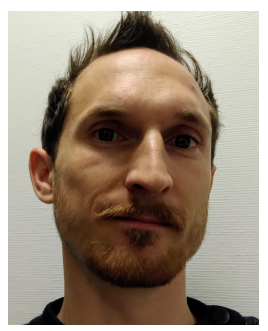

Romain Husson received the M.Sc. degree from the Supaero Engineering School, Toulouse, France, in 2009, and the Ph.D. degree in physical oceanography from the European Institute for Marine Studies (IUEM), Brest, France, in 2012, where he developed methods to estimate synthetic swell field from SAR wave mode observations at Collecte Localization Satellite (CLS).

Since 2013, he has been involved in applicative and research and development projects, such as wind/wave products for marine renewable energy. $\mathrm{He}$ also contributes to several ESA and European projects, such as $\mathrm{Cal} / \mathrm{Val}$ activities for Envisat, Sentinel-1 Mission Performance Center, and CFOSAT, as a Level-2 Expert. His general research interests include the development of new methodologies for the estimation of wind and wave products based on SAR and downstream applications. 